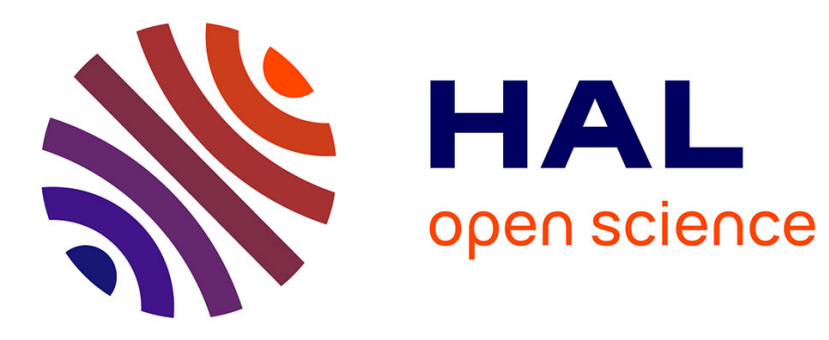

\title{
Micro-Crack Clustering, Non Local and Gradient Damage Models \\ Gilles Pijaudier-Cabot
}

\section{To cite this version:}

Gilles Pijaudier-Cabot. Micro-Crack Clustering, Non Local and Gradient Damage Models. Damage and Fracture of Disordered Materials, Springer Verlag Pubs, pp.179-215, 2005, 10.1007/978-3-70912504-5_5. hal-01007520

\section{HAL Id: hal-01007520 \\ https://hal.science/hal-01007520}

Submitted on 22 Nov 2018

HAL is a multi-disciplinary open access archive for the deposit and dissemination of scientific research documents, whether they are published or not. The documents may come from teaching and research institutions in France or abroad, or from public or private research centers.
L'archive ouverte pluridisciplinaire $\mathbf{H A L}$, est destinée au dépôt et à la diffusion de documents scientifiques de niveau recherche, publiés ou non, émanant des établissements d'enseignement et de recherche français ou étrangers, des laboratoires publics ou privés. 


\title{
MICRO-CRACK CLUSTERING, NON LOCAL AND GRADIENT DAMAGE MODELS
}

\author{
G. Pijaudier-Cabot \\ LMT-Cachan, Ecole Normale Supérieure de Cachan, Cachan, France
}

\begin{abstract}
The aim of this chapter is to show how the results from statistical analyses of damage and micro mechanics can inspire phenomenological modelling. namely continuous damage models. Non local and gradient dependent damage models are introduced. The analysis of stain localisation shows the importance of incorporating an internal length in the constitutive relations. While this internal length can be regarded as a correlation length in statistical models. Finally. these continuum models are shown to be consistent with fracture mechanics analyses of structural components.
\end{abstract}

\subsection{Introduction}

Continuous damage models are constitutive relations in which the mechanical effect of cracking and void growth is introduced with internal state variables which act on the elastic stiffness of the material (see e.g. Krajcinovic 1989 or Lemaitre and Chaboche 1989 for reviews on this topic). In most cases, continuous damage modelling is a phenomenological approach to the description of an elastic material containing voids or cracks. It is possible, however, to lay the basis of such macroscopic models on analyses of lattices and'or arrays of crachs.

From these analyses, we will see that two characteristics of damage models arise. The first one is that damage should enter in the macroscopic description as a variation of the material stiffness. The second one is that, in the course of progressive damage, initial disorder in the material tends to disappear and spatial correlation occurs.

In this chapter, we will start from such observations and see how they can be incorporated in constitutive relations. We will arrive to non local and gradient damage models and investigate, at the macroscopic level, how failure can be described as a process of localisation of damage. This study will highlight the necessity of using non local model on physical grounds since, without them, failure is predicted to occur without energy dissipation.

\subsection{Damage in a discrete system}

We are studying here a discrete system which captures the essential ingredients of the behaviour of a disordered material. It is a regular two-dimensional lattice whose bonds are one dimensional (see Fig. I-a). The lattice size is $L \times L$ where $L$ is related to the total number of bonds $n=2 L^{2}$. Each hond behaves linearly up to an assigned threshold where brittle failure is reached. The model does not aim at describing a specific material. It ought to be the simplest 
model whose thermodynamic limit (lattice of infinite size) should be described by the damage theory, as we will see further. Yet, this description includes the essential ingredients: a two dimensional geometry, initial disorder, interactions and redistribution as the number of broken bonds increases.

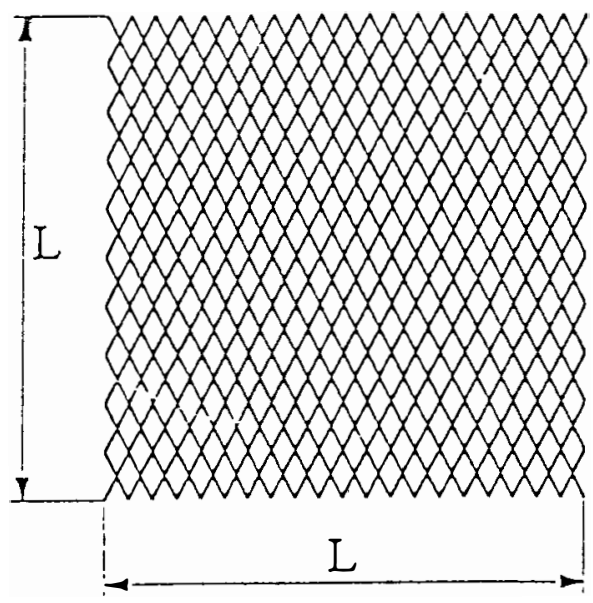

(a)

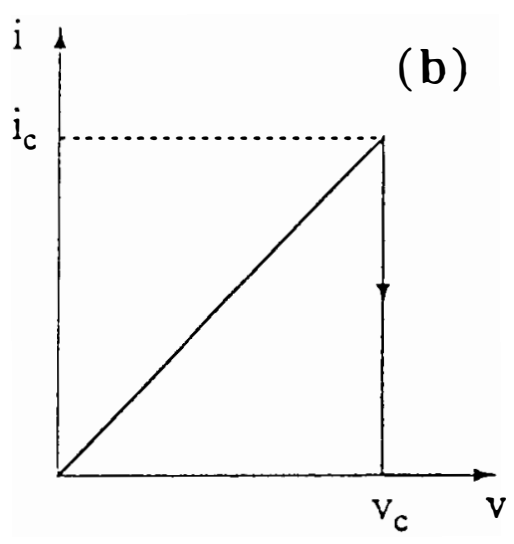

Figure 1. (a) Lattice used for the analysis; (b) behaviour of one bond.

Instead of solving a mechanical problem, we use an electrical analogy which turns out to be strictly equivalent to the mechanical problem. The equations of equilibrium are similar but one dimensional instead of being two dimensional (same as in heat conduction problems). The results can be interpreted in a mechanical fashion provided the current is replaced by the stress, the voltage by the strain and the conductance by the Young's modulus. The scaling properties of the mechanical problem and those of the electrical problem are indistinguishable (de Arcangelis and Herrmann. 1989). Figure 1-b shows the behaviour of one bond. The same conductance (stiffness) is assigned to all the bonds. The heterogeneity of the material properties is restricted to the variability of the maximum current at failure $i$. (which is equivalent to the peak stress). We have chosen here a distribution which is constant between 0 and 1 , hence it is representative of a large disorder which yields to diffuse damage which localises progressively. The boundary conditions at the limit of the lattice are periodic so that the behaviour of an infinite system is represented and boundary effects are avoided. A constant jump of voltage is applied along the two boundaries which are perpendicular to the direction $y$, and symmetry conditions are applied along the two other boundaries. At each stage of loading. a unit current $I$ (load) is applied on the lattice. The current in each bond $i$ is computed (solution of a linear algebraic system of $n / 2$ equations according to Kirchhoff law) along with the overall 
conductance of the lattice. The computational algorithm removes one bond at a time. In order to determine the next bond to be broken at a given step. We look for the bond where the ratio $i_{i} / i$ is minimum. This bond will fail when the current applied to the lattice is:

$$
I_{c}=\min \left(\frac{i_{c}}{i}\right)
$$

The voltage $V_{c}^{\prime}$ and the overall conductance of the lattice are obtained from this critical current.

Note that there are two competing effects in Eq.(1): On one side and because the material is disordered, bonds with a small threshold $i_{i}$ may fail first. On the other side, single crack propagation in the lattice produces a concentration of current (e.g. at the crack tip) which may cause a bond with a high threshold to fail. This equation illustrates how redistribution and disorder may compete during the failure process. Initially all local currents are identical, and thus the first bond to break is the weakest. If the disorder is strong enough, the enhancement of current in the vicinity of this first broken bond is not enough to induce a failure, and the second weakest bond may fail. This process yields a diffuse damage. However, the current distribution becomes broader progressively and thus bonds are expected to break in series of spatially localised clusters leading to bigger and bigger micro-cracks which are still distributed over the entire lattice. Eventually, at some stage, the current heterogeneity due to the presence of a large density of broken bonds will dominate over the distribution of strength and lead to a macroscopic localisation. A more quantitative discussion along these lines will be proposed in the sequel.

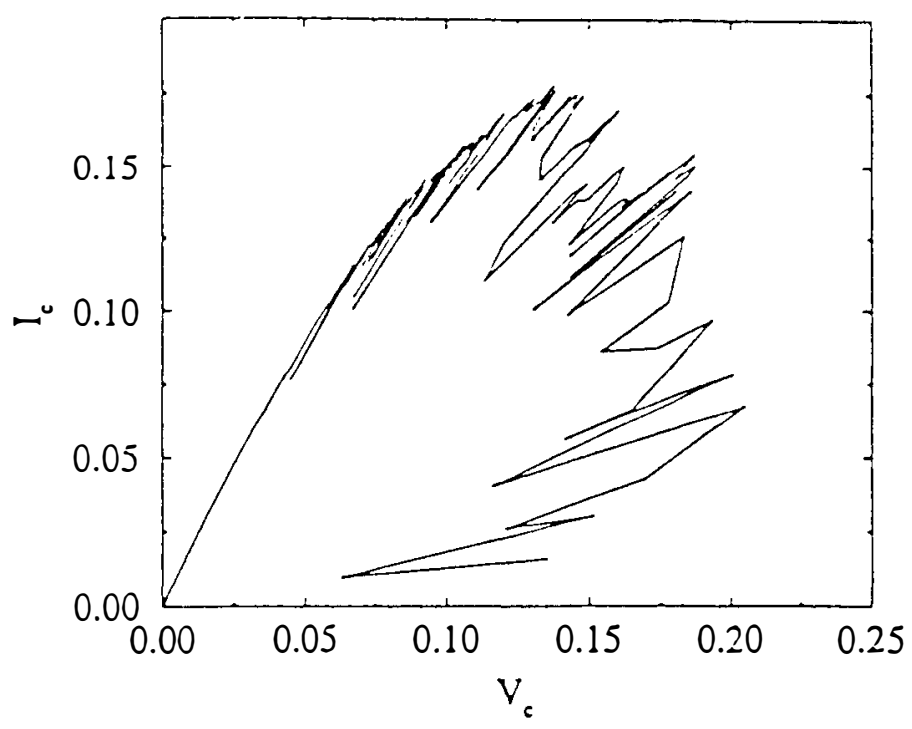

Figure 2. Cilohal response of the discrete sy stem. 
Each lattice possesses a unique distribution of bond thresholds. Obviously, analyses cannot rely on a single realisation of the random process which assigns the threshold distribution. Several computations with different random seeds must be performed and analysed in a statistical fashion. The number of runs decreases as the size of the lattice increases in order to maintain computer times which are reasonable. For instance the computation of each lattice of size $64 \times 64$ took $4 \mathrm{cpu}$ hours on a medium size workstation. Figure 2 shows one of these plots for a lattice of size $(32 \times 32)$.

The envelope of the characteristic points yields a response which is similar to that of concrete. The various snap-backs mean that under increasing voltage (strain), several bonds may break during an infinitesimal positive increment of voltage. Note that at any stage, the unloading path is linear. The equivalent mechanical lattice has a global response which agrees with continuous damage models, without damage induced inelastic strains, unless the equivalent of internal stresses are introduced in the model as in Schmittbuhl and Roux (1994). Figure 3 shows a $64 \times 64$ lattice at the inception of complete failure, when only one bond remains unbroken (i.e. when the lattice is about to separate into two pieces). As the number of broken bonds increases, their location localises over a rough line progressively - damage is diffuse at the beginning of the failure process and localises to form a macro crack. It is important to note that this localisation concerns the incremental damage. The final aspect of the distribution of micro cracks will appear as diffuse since it incorporates micro cracks generated at all stages.

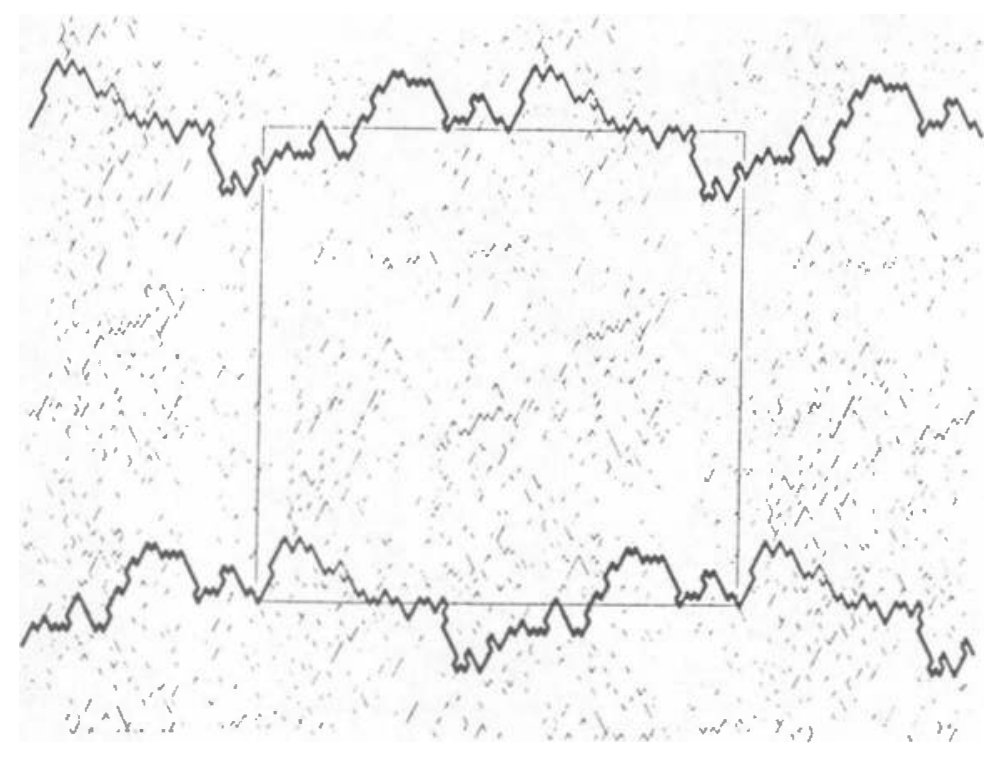

Figure 3. Lattice just before complete failure.

The numbers of broken bonds at peak and at failure are interesting because they show that damage is distributed. Would damage be localised along a single line (as for brittle materials), 
the number of broken bonds at failure would be twice the system size, i.e. the number of bonds along a straight line in the $x$ direction, and the number of broken bonds at peak would be zero. This result would be achieved when there is no disorder in the lattice. Table 1 shows for each different size of lattice considered the number of runs performed, the averaged number of broken bonds at the peak current (load) $n_{p}$ and the averaged number of broken bonds at failure $n_{c}$. It should be noted that the number of broken bonds at failure is always higher than the number of broken bonds at peak. This indicates that the failure process is rather progressive and that the behaviour of the lattice departs from a brittle response due to spatial correlation and local disorder.

\begin{tabular}{llll}
$L$ & Runs & $n_{p}$ & $n_{c}$ \\
\hline 8 & 1000 & 19 & 29 \\
16 & 500 & 76 & 105 \\
24 & 250 & 149 & 198 \\
32 & 250 & 255 & 340 \\
64 & 30 & 947 & 1148 \\
\hline
\end{tabular}

Table 1. Number of broken bonds at peak and at failure as a function of the lattice size.

First, we are interested in the evolution of damage in the lattice and its influence on the global properties. For this, we will look at the distribution of the current in the discrete model at several states of damage. Second, we expect to observe a correlation length which characterises the smallest representative volume element at a given state of damage. This correlation length appears in the spatial distribution of currents and consequently in the spatial distribution of broken bonds. Both aspects will be examined in the next sections.

\subsubsection{Global properties of the discrete model}

The global properties of the discrete model at any state of damage are, in this approach. directly connected to the local distribution of current $N(i)$. Instead of characterising the distribution itself, valuable information can be obtained with the analysis of the moments of the distribution of the current. The moment of order $m$ is defined as:

$$
M_{m}=\int i^{m} N(i) d i
$$

Here, we will limit the analysis to moment of order up to 4 . This assumption is equivalent to a truncature in a series development. These moments are of interest because of their physical meaning : the moment of order zero is the number of unbroken bonds. The first order moment is related to the average current, the second order moment is proportional to the overall conductance $G$ : 


$$
M_{2}=\int r^{2} N(i) d i=\underline{2} G V^{2}=2 G
$$

where $r$ is the local resistance of the bond (unit resistance here) and $V$ is the global voltage jump applied to the lattice, equal to one in our case. Note that the fourth order moment is a measure of the dispersion of conductance.

It is natural to investigate whether the number of broken bonds is a variable which characterises correctly' the evolution of damage in the continuum sense, that is a degradation of the global conductance or stiffness. If the number of broken bonds $q / L^{2}$ (divided by the dimension of the lattice in order to have a quantity which is size independent) is the pertinent damage variable, the plots of the global conductance versus the non dimensional number of broken elements should be independent of the size of the discrete model. Delaplace et al. (1996) have shown that this is not true. Figure 4 shows a log-log plot of the moments as a function of the second order moment for different sizes of lattice. These plots do not depend on the size of the system, at least before the peak is reached. Hence, damage can be defined as the variation of the overall conductance during failure. This variable describes well the distribution of the current (stress) in a size independent fashion. A similar observation has been reported by Krajcinovic and Basista (1991). As we will see further, it will be used in the derivation of phenomenological damage models.

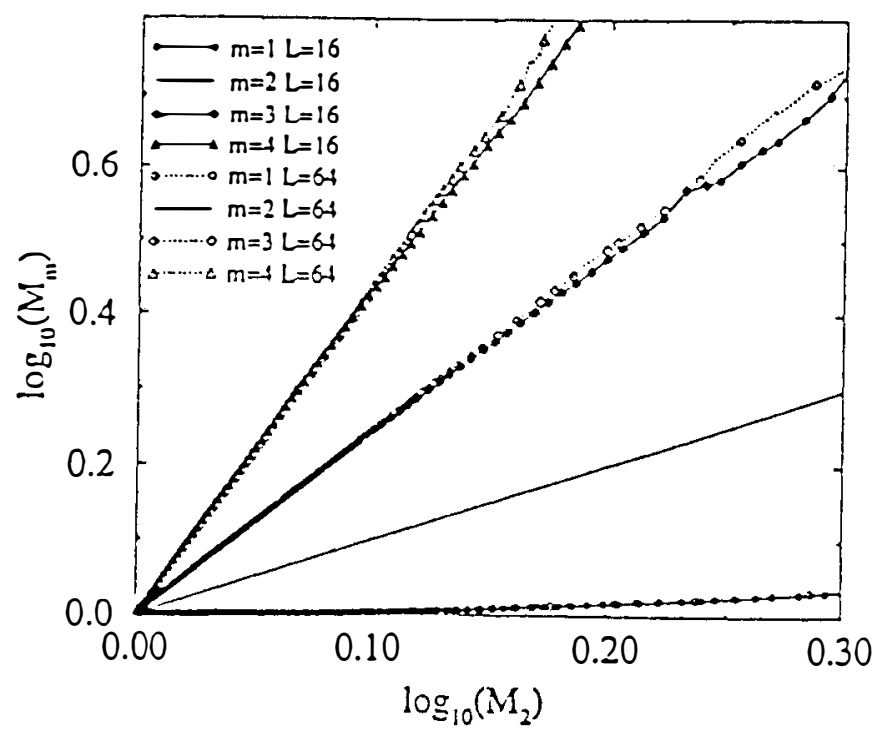

Figure 4. Evolution of the first four moments of the distribution of current as a function of the overall conductance. 


\subsubsection{Existence of a correlation length}

Assume that there is a length denoted as $\xi$ which defines the smallest size of the representative volume of the material (RVE). By construction, this length is smaller or equal to the size of the lattice. This quantity is a correlation length in a statistical sense. Above this size, the material can be regarded as homogeneous, i.e. without correlation. The local response of each bond in the discrete model is not independent for cells in the lattice bellow this size. In such cells, the response cannot be regarded as that of an homogeneous continuum. In order to exhibit the existence of a correlation length as the number of broken bonds increases, one can analyse their distribution over the cell. This analysis is based on the variation of distance between two bonds in the lattice which are consecutively broken during the loading history according to the foregoing definitions: the distance between to neighbouring vertical bonds is 1 because there are $L$ bonds on each column of the cell, and the distance between to neighbouring horizontal bonds is 0.5 because there are $2 L$ bonds on each row of the cell.

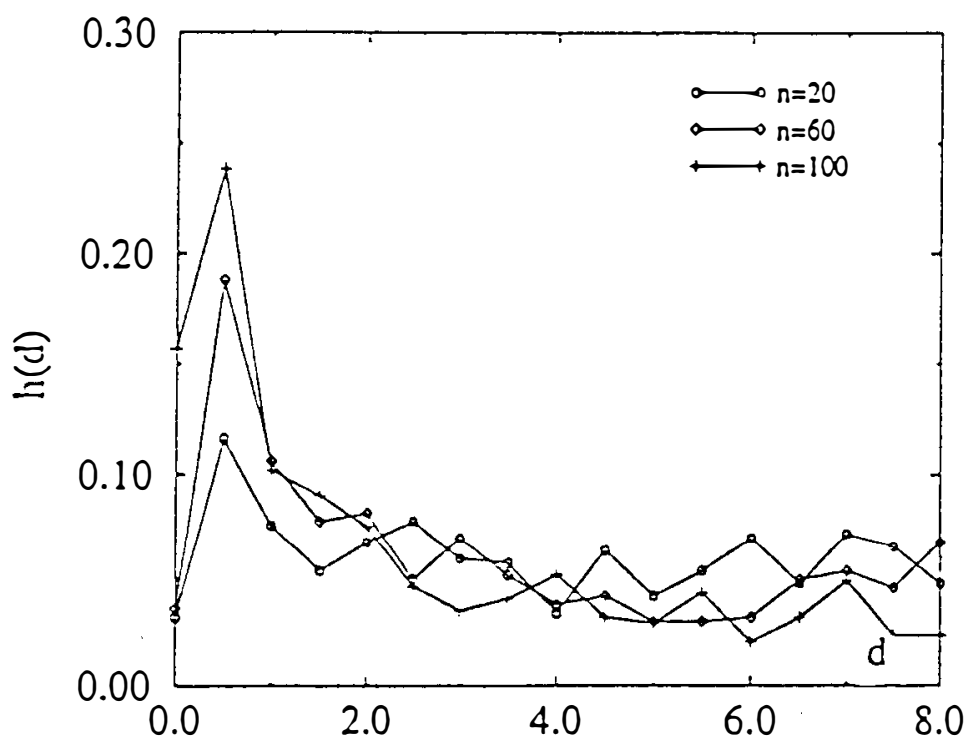

Figure 5. Histograms of the distribution of horizontal distance.

Figure 5 show's for systems of size $16 \times 16$ the histograms of the distribution of horizontal distance between two consecutive broken bonds denoted as $h(d)$ at the beginning of damage (20 broken bonds). near the peak ( 60 broken bonds) and near failure ( 100 broken bonds). In the absence of a correlation length, these histograms should be a set of horizontal lines, which means that the next broken bond can found anywhere in the lattice. These histograms can be approximated at least up to the peak: the distribution of the broken links is assumed to follow 
a power law function of the distance up to $\xi$ and then it is an horizontal line. The length $\xi$ corresponds then exactly to the smallest size of the RVE:

$$
h(d) \approx d^{-\tau} \text { for } \mathrm{d} \leq \xi, \mathrm{h}(\mathrm{d})=\mathrm{h}^{*} \text { for } d \in\left[\xi \cdot \frac{\mathrm{L}}{2}\right]
$$

The value of exponent $\tau$ is 0.67 for the normalised distributions shown on figure 5 . The distance $\xi$ is also the length at which the distribution changes from diffuse damage (constant distribution) to a progressive localisation of damage. Hence, it can be considered as a measure of the size of the zone in which damage localises. The first order moment of the distribution $h(d)$ can be obtained numerically. Substitution of Eq. (4) in the expression of the first order moment yields an equation where the only remaining unknown is the correlation length.

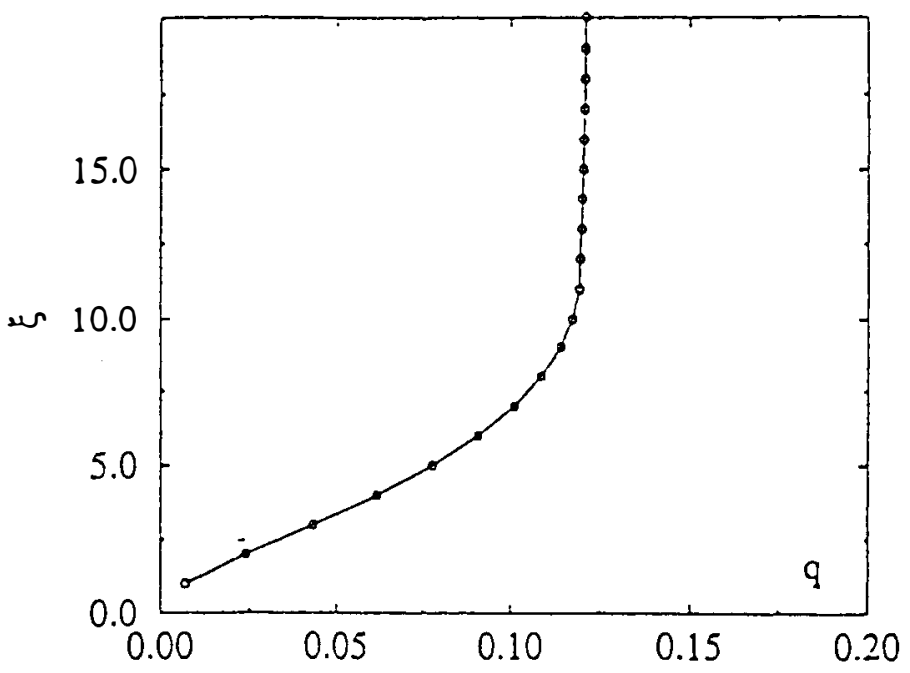

Figure 6. Internal. correlation length vs. number of broken bonds.

Figure 6 shows the evolution of this correlation length. It increases with increasing damage. The existence of a correlation length suggests that the damage process should be spatially correlated. In section 4 , we will consider the deterministic case of a array of micro cracks and show again that some spatial correlation exists in the development of damage. 


\subsection{Continuous Damage}

As concluded in the previous section, the influence of damage on the response of the material is a degradation of its elastic stiffness. The stress-strain relation reads:

$$
\sigma_{i j}=E_{i j k l}^{\text {clamaged }} \varepsilon_{k l}
$$

where $\sigma_{i j}$ is the stress component, $\varepsilon_{k l}$ the strain component, and $E_{i j k l}^{\text {clamaged }}$ is the stiffness coefficient of the damaged material. We will focus attention on the simple, isotropic, scalar damage model because it is the most widely damage model employed and also because it has been proved to be an efficient model for the description of the tensile failure of concrete and reinforced concrete structural components (Mazars 1984, Mazars and Pijaudier-Cabot 1989).

The stress strain relation of an isotropic damaged elastic material contains two damage variables $d$ and $\delta$ :

$$
\varepsilon_{i j}=\frac{3}{2 E_{0}(1-d)}\left(\sigma_{i j}-\frac{\sigma_{k k}}{3} \delta_{i j}\right)+\frac{\left(1-2 v_{0}\right)}{2 E_{0}(1-\delta)}\left[\sigma_{k k} \delta_{i j}-\sigma_{i j}\right]
$$

$E_{0}, v_{0}$ are the Young's modulus and Poisson's ratio of the undamaged isotropic material respectively, and $\delta_{i j}$ is the kronecker symbol. The damage variables $d$ and $\delta$ are equal to 0 initially (for the undamaged material). At complete failure, the elastic stiffness of the material vanishes and the two variables are equal to 1 . The subsequent assumption $d=\delta$ yields the stress-strain relationship:

$$
\varepsilon_{i j}=\frac{1+v_{0}}{E_{0}(1-d)} \sigma_{i j}-\frac{v_{0}}{E_{0}(1-d)}\left[\sigma_{k k} \delta_{i j}\right]
$$

According to this equation, the Poisson's ratio of the material is not affected by damage. The elastic (i.e. free) energy per unit mass of material is:

$$
\rho \psi=\frac{1}{2}(1-d) \varepsilon_{i j} E_{i j k l}^{0} \varepsilon_{k l}
$$

where $E^{0}$ is the stiffness tensor of the undamaged material. This energy is assumed to be the state potential, which means that the thermodynamic forces associated to the two variables describing the material, the strain tensor and the damage variable, derive from this potential: 


$$
\begin{aligned}
& \sigma_{i j}=\frac{\partial \rho \psi}{\partial \varepsilon_{i j}} \\
& Y=-\frac{\partial \rho \psi}{\partial d}=\frac{1}{2} \varepsilon_{i j} E_{i j k l}^{0} \varepsilon_{k l}
\end{aligned}
$$

Note that Eq. (9-a) is exactly the same as Eq. (7). $Y$ is the damage energy release rate. The meaning of this variable is deduced from the calculation of the energy dissipation. The dissipation rate is the difference between the total (internal) variation of mechanical energy for any arbitrary rate of strain and the elastic (recoverable) energy rate:

$$
\dot{\phi}=\sigma_{i j} \dot{\varepsilon}_{i j}-\rho \dot{\psi}
$$

Substitution of Eqs (7-9) yields the dissipation rate $\dot{\phi}$ as a function of the damage variable:

$$
\dot{\phi}=Y \dot{d}
$$

Therefore, $Y$ is the energy released per unit rate of damage. In this constitutive relation (with a single damage variable), the second principle of thermodynamics constrains the time derivative of damage to be zero or positive. Damage cannot decrease because the rate of dissipation cannot decrease according to the Clausius-Duhem inequality.

The evolution of damage requires the definition of a loading function and an evolution law which can be similar to the evolution equation in associated plasticity. The constitutive relation is sometimes given in an integrated format. We will see in section 5 two examples of such constitutive relations.

\subsection{Micro Cracking and Continuous Damage}

In most experiments, say a direct tension test or a bending test (Mazars, 1984), damage starts as a uniform distribution of propagating micro cracks. At the inception of failure, crack propagation is localised into a narrow zone and only a few micro cracks propagate to form the visible macro crack at failure (Chudnovsky et al., 1989). In order to provide some justifications for damage and non local damage models, Pijaudier-Cabot and Berthaud (1990) have considered the two problems shown on figure 7 which represent : (1) the initial state of damage, i.e. a regular array of cracks in an elastic matrix and (2) the situation in which one crack has propagated while all the others crack of the array remain stable. This last situation is meant to simulate the inception of failure with damage localisation in a simplistic (qualitative), one-dimensional fashion.

\subsubsection{Fracture Mechanics Analysis}

Consider the regular crack array shown on figure 7-a. This array is subjected to a remote tensile loading denoted as $\sigma_{\infty}$. The objective is to compute the stress intensity factor and energy 
release for each crack tip and to exhibit the effect of crack interaction. For this, an approximate method, originally proposed by Kachanov (1987) is implemented. First, we use the superposition and replace the tensile loading $\sigma_{\infty}$ by an internal pressure $-\sigma_{\infty}$ in each crack $\mathrm{S}_{\mathrm{i}}$. Then, this equivalent problem of $\mathrm{N}$ interacting cracks is replaced by $\mathrm{N}$ problems in which each crack is considered alone in an infinite medium and is subjected to an unknown intermal pressure $P_{i}\left(x_{i}\right)$ given by Eq. $(12)$ :

$$
P_{i}\left(x_{i}\right)=-\sigma_{\infty}+\sum_{j=1 . j \neq i}^{N} p_{j i}\left(x_{i}\right)
$$

where $p_{j i}\left(x_{i}\right)$ represents the effect of the crack $\mathrm{S}_{\mathrm{j}}$ on the crack $\mathrm{S}_{\mathrm{i}}$.
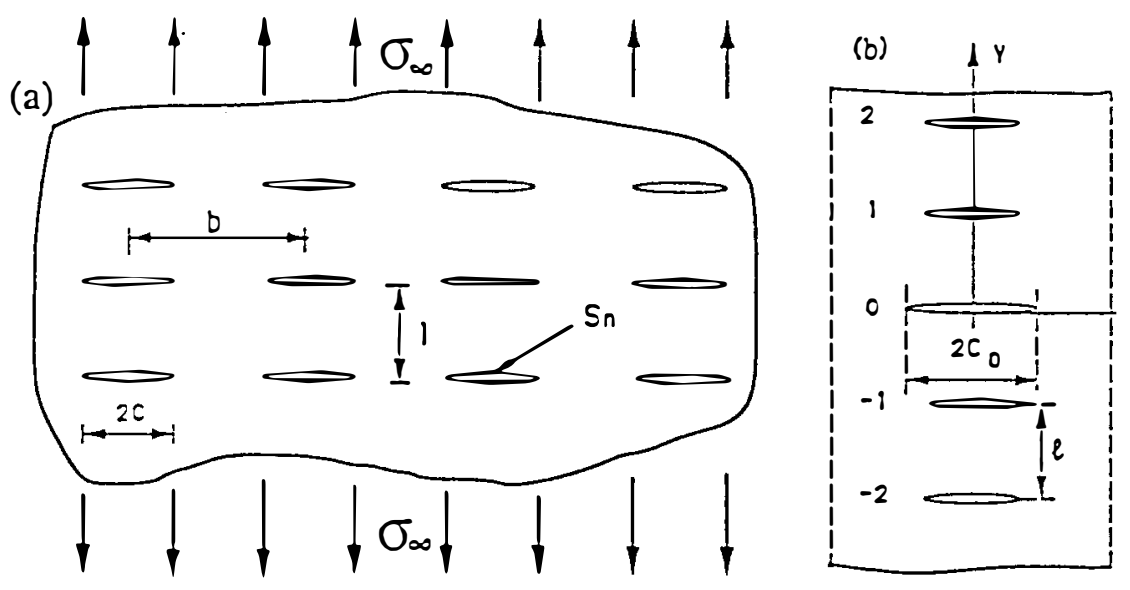

Figure 7. Micro cracked model material: (a) uniform crack density; (b) inception of localisation.

The unknown pressure $P_{i}\left(x_{i}\right)$ is expanded in a series of base functions which are Legendre polynomials:

$$
P_{i}\left(x_{i}\right)=\sum_{r=0}^{R} a_{r}^{i} L_{r}^{i}\left(x_{i}\right)
$$

The maximum order of the expansion is denoted $\mathrm{R}$ and $a_{r}^{i}$ are the coefficients of the expansion where i refers to the cracks $S_{i}$ and $r$ refers to the considered order of expansion. It is also natural to expand the interaction terms $p_{j i}$ of the $\mathrm{S}_{\mathrm{j}}$ crack on the $\mathrm{S}_{\mathrm{i}}$ crack: 


$$
p_{j i}\left(x_{i}\right)=\sum_{r=0}^{R} a_{r}^{j} f_{j i}^{r}\left(x_{i}\right)
$$

In this equation, the function $f_{j i}^{r}$ represents the stress field on the imaginary location of the crack $\mathrm{S}_{\mathrm{i}}$ due to the crack $\mathrm{S}_{\mathrm{j}}$ loaded by a pressure distribution given by $L_{r}^{j}$.

The coefficients $a_{r}^{i}$ are the solution of the linear algebraic system which is obtained from Eq.(13) for $\mathrm{i}=1, \mathrm{~N}$ using the orthogonality properties of Legendre polynomials. The stress intensity factor is approximated using the second term of Eq.(13). In the present examples, we took $R=2$ which provides a sufficient accuracy.

\subsubsection{Homogenisation - Relationship with Continuous Damage}

The aim is here to evaluate the macroscopic stiffness of the micro cracked medium depicted above, in the direction which is orthogonal to the crack planes. Obviously, the micro cracked material which is considered is not isotropic because the crack orientation is not random but periodic. Therefore, the results established in this section are only qualitative.

The elastic energy in each cell is computed and a simple equivalence with an elastic continuum is assumed. The elastic energy in each cell of the micro cracked body is:

$$
W=\frac{1}{2} \int_{-h / 2}^{h / 2} \int_{-l / 2}^{l / 2} \sigma(x, y): E^{-1}: \sigma(x, y) d y d x
$$

where $E^{-1}$ is the compliance matrix of the elastic, uncracked, material. In the equivalent homogeneous material, the average elastic strain due to the crack opening in each cell is:

$$
\gamma=\frac{1}{b l} \frac{\partial W}{\partial \sigma_{\infty}}
$$

The total strain in each cell is then the sum of the deformation of the material and of the average strain due to crack opening. This deformation is totally reversible, provided the crack lengths in each cell remain constant. It follows the equalities (see Bazant, 1987):

$$
\varepsilon=\gamma+\frac{\sigma_{\infty}}{E_{0}}, \quad \varepsilon=\frac{\sigma_{\infty}}{E}=\frac{\sigma_{\infty}}{E_{0}(1-d)}
$$

where $E_{0}$ is the directional stiffness of the material without cracks and $E$ is the directional stiffness of the material with cracks. This stiffness is set proportional to $E_{0}$ and it is a function of the damage variable. Eqs.(15-17) provide an expression of the damage variable $d$ as a function of the crack density $\beta$ and of the interaction between cracks: 


$$
d=\frac{H \beta}{1+H \beta}, \quad \beta=\frac{c^{2}}{l b}
$$

$H$ is the interaction parameter which depends on the crack configuration. For the plane-strain mode I problem where the crack lengths are equal in each cell, this interaction parameter is:

$$
H=2 \pi\left(1-v^{2}\right)\left(a_{0}-\frac{a_{2}}{8}\right)
$$

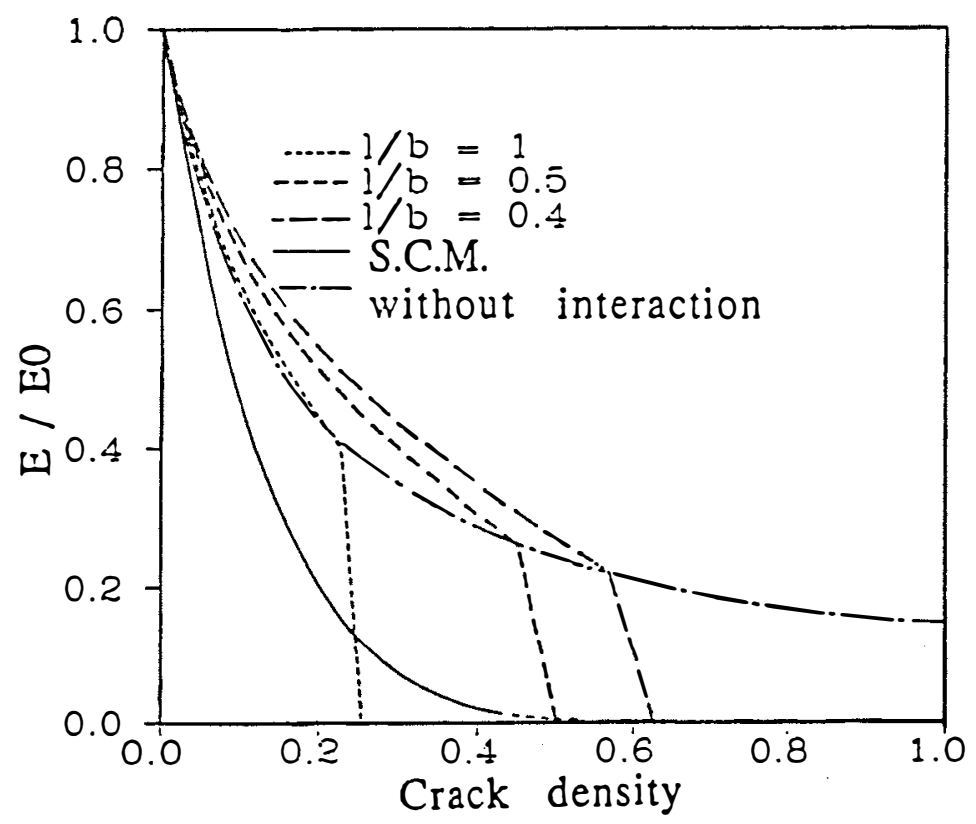

Figure 8. Evolution of the elastic modulus with crack density.

In this case the coefficients $a_{r}^{i}$ (here $\left.a_{0}, c_{2}\right)$ are equal from one cell to another. They are solution of a $2 \times 2$ linear algebraic system of equations which is solved independently from the intensity of the remote stress $\sigma_{\infty}$. Eq.(18) highlights the effect of the interactions between cracks and of the crack density on the equivalent stiffness of the micro cracked medium. The evolution of the elastic modulus $E$ is plotted on figure 8 for different crack spacings $b$ and $l$. The theoretical prediction obtained with a Self Consistent Method (SCM) (Laws and Brockenbrough, 1987) is also plotted. Compared to SCM, the present approach shows that interactions produce first a shielding effect for low crack density, and an amplification effect for 
large crack densities. This amplification results in a stiffness which is much lower than the one calculated with SCM in the case of a square cell.

For a dilute distribution of micro cracks (small crack density), the two models are similar. They are consistent with the lattice analysis in the regime controlled by the initial disorder. We will see next that interaction may play a central role when the geometry of distributed cracking changes, same as in lattice analysis when the damage process start to exhibits a non zero correlation length.

\subsubsection{Crack Interaction and Non Local Damage}

Consider again the elastic body with cracks shown on figure 7. When the crack density is low (i.e. $b / c \geq 1$ ), the interactions between collinear cracks can be neglected and we can consider only parallel cracks which represent the first stage of damage in a tensile loading test. The inception of localisation in that crack array is described by the case where one of the cracks has propagated symmetrically (Fig. 7-b). We are going to compute the new value of the damage variable corresponding to this crack configuration and to compare with the previous analysis qualitatively. For the purpose of this demonstration, the crack propagation conditions are not considered. We will compare two states of damage very close to each other.

Crack interaction appears naturally in the homogenisation as a non local effect. The crack opening in one cell is affected by the state of deformation and cracking in the other cells. In the parallel crack system shown on figure 7-b, the crack length in cell 0 has been perturbed of a small quantity $\delta c$. The strain energy in a given cell $k$ can be approximated in plane strain by:

$$
\begin{aligned}
& W_{k}=\frac{\sigma_{\infty} P_{k}\left(1-v^{2}\right) c_{k}}{E_{0}}+ \\
& \frac{1}{2}\left[\int_{-h / 2}^{h / 2} \sigma(x,-l / 2) \cdot \vec{n} \cdot \vec{u}(x,-l / 2) d x+\int_{-h / 2}^{h / 2} \sigma(x, l / 2) \cdot \vec{n} \cdot \vec{u}(x, l / 2) d x\right]
\end{aligned}
$$

In this expression, the second and third terms represent the flow of energy through the contour of each cell $k$ due to the interaction. These are typically non local terms which appear in all cells, including those where the crack length does not change. According to the homogenisation method, the influence of this perturbation will produce a variation of damage in those cells although the crack length has not changed. Damage must not only be a function of the crack density in each cell but also of the crack densities in neighbouring cells. Then, the elastic energy in an equivalent homogenised material should not be a function of the overall strain and damage variable only. It should include quantities which refer to the crack or damage distribution. One possibility for this is to define damage, denoted now as $D$, in the equivalent, homogenised, material as a weighted volume average:

$$
D(x)=\int_{V} \psi(x-s) d(s) d s
$$


$D$ is called the "non local damage variable" and $d$ is the "local damage variable" by" reference to the terminology used in non local elasticity (Eringen and Edelen, 1972). The weight function $\psi$ is normalised so that

$$
\int_{V^{\prime}} \psi(s) d s=1
$$

It follows from this definition that when the local damage is homogeneous over an infinite body, it is equal to the non local damage. Therefore, $d(x)$ is equal to the value of damage given in Eq.(18) calculated in a fictitious medium which has a constant crack density equal to $\beta(x)$. This is the reason why $d(x)$ is still called a damage variable. It is a local quantity defined at each material point independently from the distribution of cracking in the structure considered.

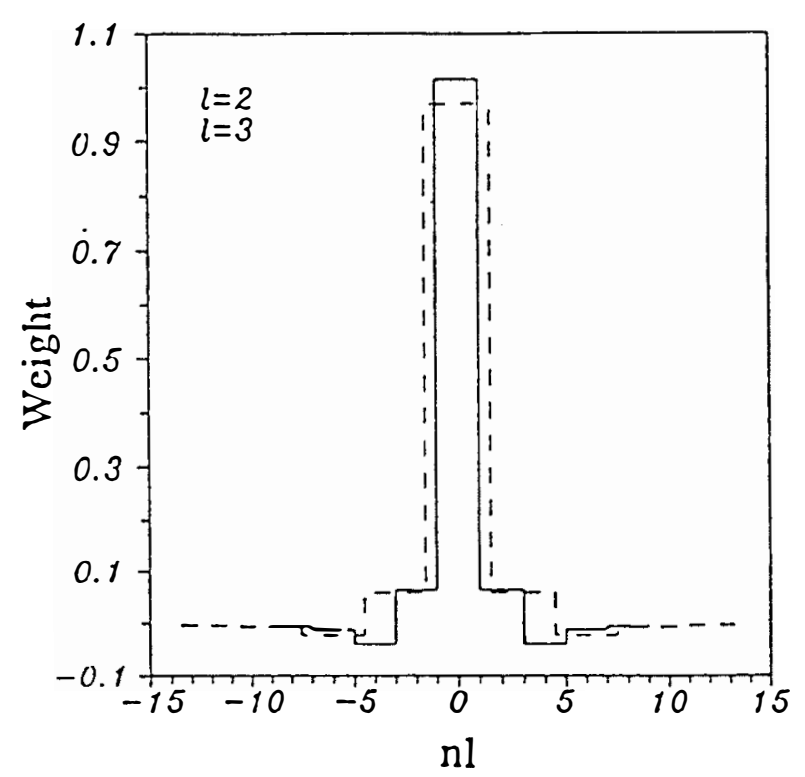

Figure 9. Weight function at the inception of localisation.

With these definitions, the weight function in Eq. (21) can be calculated: the value of the macroscopic non local damage $D_{k}$ in each cell $k$ is computed using Eqs. (16-17) in which the non local damage is substituted to the local damage. The definition of the local damage provides also the expression $d_{m}$ in each cell $\mathrm{m}$. Its value in cell $\mathrm{m}$ is computed by considering that cell $\mathrm{m}$ is sitting in a fictitious medium of constant crack density equal to that in cell $\mathrm{m}$. In each cell, the local and non local damage are different because the perturbation of the crack length produces a modification of the crack interactions. Eq. (21) is rewritten as a discrete sum: 


$$
D_{k}=\sum_{m=-\infty}^{m=+\infty} \bar{\psi}_{k m} d_{m}
$$

where $\bar{\psi}_{k m}$ is the mean value of $\psi$ over the cell $\mathrm{m}$ when $D$ is calculated is the cell $\mathrm{k}$.

Since the values of local and non local damage are known everywhere, the weight function may easily be deduced. Figure 9 shows this weight function for two crack spacings $l=2 c$ and $\lceil=3 c$. This plot shows also that damage will grow in cells number $l$ and $-l$, which are adjacent to the cell 0 in which the crack propagates, despite the length of the cracks in these cells remains constant.

Would the interactions remain similar during failure, the variable which describes the effect of micro cracking on the elastic constants would be purely local. Non locality is caused by a change of interactions during progressive failure. These results show also that the weight function, which is the mathematical representation of the variations of crack interaction, is a function of the micro crack length and of the state of strain (or stress). In the non local damage models, this weight function will be assumed to remain constant. Furthermore, the weight distribution will be assumed to be spherically symmetric. A constant weight function is an approximation of the average variations of interactions during a specific failure mode (tension in the present applications). According to homogenisation, different weight functions should be employed for different failure modes at least.

\subsection{Non Local Damage Models}

Fracture mechanics and crack interaction considerations have shown that defining damage as a weighted average seems more appropriate, especially with regard to the description of localisation of cracking in an elastic material containing a distribution of micro cracks. A non local variable enables the description of micro structural changes, (and more particularly deterministic interactions) that a local variable cannot accommodate. This result is also consistent with lattice analysis, as observed in section 1. Such a phenomenological model has been investigated by Bazant and Pijaudier-Cabot (1988), along with a constitutive relation where the variable which controls damage is averaged, instead of averaging damage itself.

Scalar non local damage models are presented in this section. Similar developments could be performed starting from a plasticity model or with an anisotropic damage model (Valanis 1991).

\subsubsection{Energy-based model}

The constitutive relation is exactly the same as for the local damage model (Eq. 7). The evolution of damage is different. The growth of damage is defined by a loading function $f$ :

$$
f(\bar{y}, D)=\int_{0}^{\bar{y}} F(z) d z-D
$$


where $F$ is (in this chapter) a function of the strain tensor which is deduced from experimental data. Instead of depending on the local energy release rate $y(x)$, the loading function depends on $\bar{y}(x)$, the average energy release rate due to damage at point $x$ of the solid:

$$
\bar{y}(x)=\frac{1}{V_{r}(x)} \int_{V^{\prime}} \psi(x-s) y(s) d s \text { and } V_{r}(x)=\int_{V^{\prime}} \psi(x-s) d s
$$

$V$ is the volume of the solid, and $y(s)$ is the energy release rate due to damage at point $s$ defined by:

$$
y(s)=\frac{1}{2} \varepsilon(s): E: \varepsilon(s)
$$

$\psi(x-s)$ is the weight function :

$$
\psi(x-s)=\psi_{0} \exp \left(-\frac{\|x-s\|^{2}}{2 l_{c}^{2}}\right)
$$

$l_{c}$ is the internal length of the non local continuum and $\psi_{0}$ is a normalising factor. This factor is such that for an infinite body $V_{r}(x)=1$. The internal length of the non local continuum depends on the size of the heterogeneities in the material. An usual approximation of this length is $l_{c} \approx 3 d_{a}$ where $d_{a}$ is the maximum size of the aggregate in concrete (Bazant and Pijaudier-Cabot, 1989).

The evolution law is prescribed as in. plasticity models:

$$
\dot{D}=\delta \frac{\partial g}{\partial \underline{y}}
$$

with the Kuhn-Tucker conditions $\delta \geq 0, f \leq 0$ and $\delta f=0$. The function $g$ is the evolution potential controlling the growth of damage and $\delta$ is here the damage multiplier. In the following, we have set $g=\bar{y}$. For concrete in tension, the evolution of damage is:

$$
F(\bar{y})=\frac{b_{1}+2 b_{2}\left(\bar{y}-Y_{0}\right)}{\left[1+b_{1}\left(\bar{y}-Y_{0}\right)+b_{2}\left(\bar{y}-Y_{0}\right)^{2}\right]^{2}}
$$

with the constants $b_{1}=605 M P a^{-1}, b_{2}=5.2+10^{+} M P a^{-1}, Y_{0}=6010^{-6} M P a$. 


\subsubsection{Strain-Based Model}

This model is the non local extension of the constitutive relation due to Mazars (1984). The positive strains control the growth of damage which is mainly due to micro crack opening in mode I. The following norm called equivalent strain is defined :

$$
\tilde{\varepsilon}=\sqrt{\sum_{i=1}^{3}\left(\left\langle\varepsilon_{i}\right\rangle_{+}\right)^{2}}
$$

where $\left\langle\varepsilon_{i}\right\rangle_{+}=0$ if $\varepsilon_{i}<0$ and $\left\langle\varepsilon_{i}\right\rangle_{+}=\varepsilon_{i}$ if $\varepsilon_{i} \geq 0$; and $\varepsilon_{i}(i \in[1,3])$ are the principal strains. The non local variable $\bar{\varepsilon}$, which represents the average of the equivalent strain over the representative volume surrounding each point in the material, is the variable that controls the growth of damage (Saouridis, 1988)

$$
\bar{\varepsilon}(x)=\frac{1}{V_{r}(x)} \int_{V} \psi(x-s) \tilde{\varepsilon}(s) d s
$$

$\mathrm{V}$ is the volume of the structure, $V_{r}(x)$ is the representative volume at point $\mathrm{x}$, and $\psi(x-s)$ is the weight function, the same as for the previous model. The evolution of damage is specified according to following conditions:

$$
\begin{aligned}
& f(\bar{\varepsilon})=\bar{\varepsilon}-\kappa \\
& \text { and } \\
& \text { if } f(\bar{\varepsilon})=0 \text { and } \dot{f}(\bar{\varepsilon})=0 \text { then } D=F(\bar{\varepsilon}) \\
& \text { if } f(\bar{\varepsilon})<0 \text { or if } f(\bar{\varepsilon})=0 \text { and } \dot{f}(\bar{\varepsilon})<0 \text { then } \dot{D}=0
\end{aligned}
$$

Equations (33) define the damage surface and the growth of damage. $\kappa$ is the softening parameter and takes the largest value of $\bar{\varepsilon}$ ever reached during the previous loading history at a given time and at the considered point in the medium. Initially $\kappa=\kappa_{0}$, where $\kappa_{0}$ is the threshold of damage. $\kappa_{0}$ is the tensile strain at which damage is initiated, that is when the maximum tensile stress $f_{t}$ is reached in a uniaxial test.

The evolution law, denoted as $F(\bar{\varepsilon})$ in Eq.(33) is a function of the strain, instead of the strain rate. It is integrated with respect to time so that damage can be directly computed at any state of deformation. Damage is decomposed into two parts, $D_{t}$ for tension and $D_{c}$ for compression. $D$ is computed from the relation (34) which combines these two types of damage:

$$
D=\alpha_{t} D_{t}+\alpha_{i} D_{c}
$$



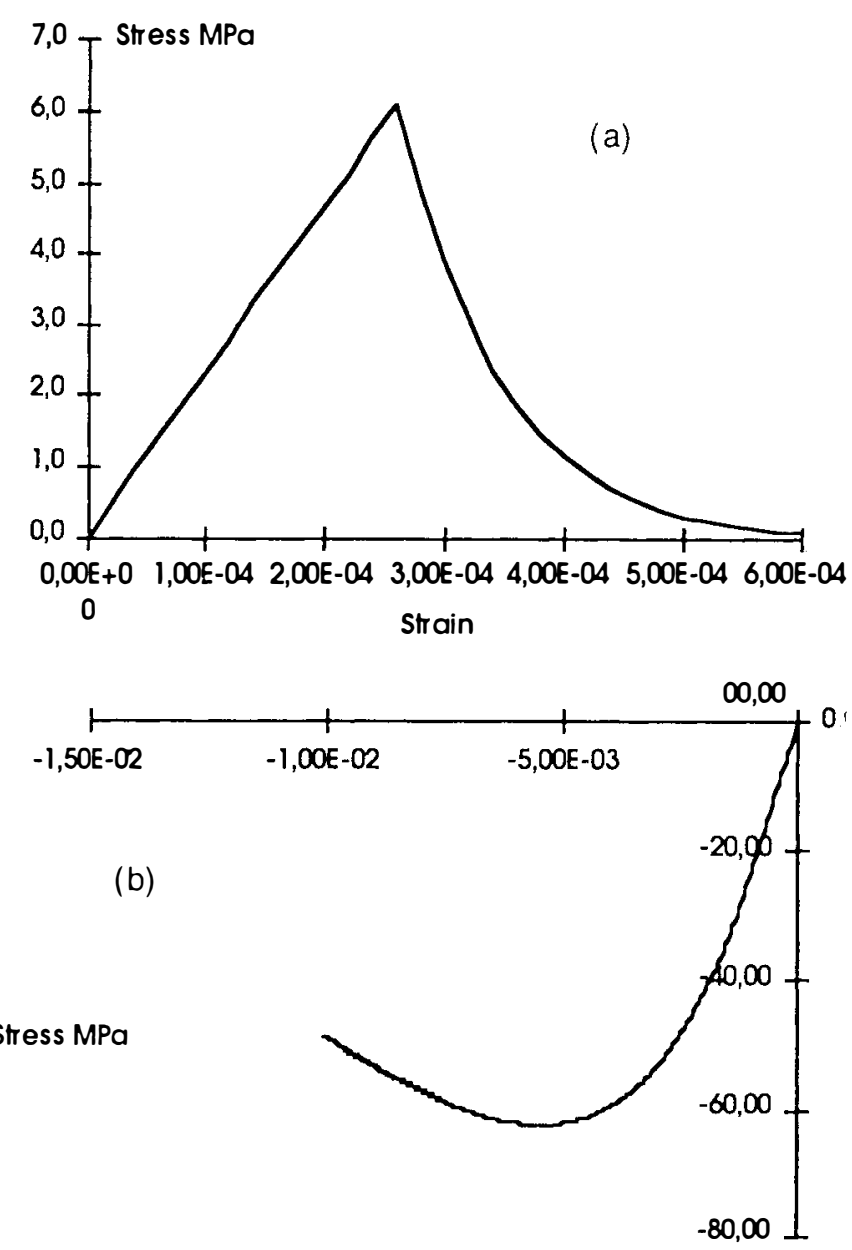

Strain

Figure 10 : Response of the Strain-Based Model in Tension (a) and Compression (b).

The factors $\alpha_{t}$ and $\alpha_{i}$ are such that in uniaxial tension $\alpha_{t}=1, \alpha_{i}=0$, and $D=D_{t}$, and in compression $\alpha_{t}=(), \alpha_{c}=1$, and $\left.l\right)=D_{i}$. The damage variables are functions of the average equivalent strain:

$$
\begin{aligned}
& I_{t}=1-\frac{\kappa_{0}\left(1-A_{t}\right)}{\bar{\varepsilon}}-\frac{A_{t}}{\exp \left(-B_{t}\left(\bar{\varepsilon}-\kappa_{0}\right)\right)} \\
& I_{c}=1-\frac{\kappa_{0}\left(1-A_{c}\right)}{\bar{\varepsilon}}-\frac{A_{t}}{\exp \left(-B_{i}\left(\bar{\varepsilon}-\kappa_{0}\right)\right)}
\end{aligned}
$$


and constants $A_{c}, B_{c}, A_{t}, B_{t}$ are model parameters. These coefficients characterise the hardening/softening response of concrete. The factors $\alpha_{t}$ and $\alpha_{c}$ are expressed as non dimensional functions of the principal strains. Note that in this model, the condition that damage cannot decrease should be added to the loading function in Eq. (33) because the factors $\alpha_{t}$ and $\alpha_{c}$ may vary in the reversible regime and could yield a decrease of damage which is inconsistent with the second principle of thermodynamics.

Figure 10 shows the response of this model in tension and in compression. The model parameters are:

$$
\begin{aligned}
& E_{0}=23400 M P a, v_{0}=0.2, \kappa_{0}=2.610^{-4}, \beta=1.05 \\
& A_{t}=1 ., B_{t}=15000, A_{c}=1.2, B_{c}=649
\end{aligned}
$$

More details on this constitutive relation, its finite element implementation, and comparisons with experimental data can be found in the works of Mazars (1984), Saouridis (1988), Mazars and Pijaudier-Cabot (1989).

\subsection{Gradient Damage Model}

Non local constitutive relations can be considered as the point of departure of gradient models. Following the approach of section 5.2, the definition of the non local effective strain in Eq. (31) can be written as:

$$
\bar{\varepsilon}=\tilde{\varepsilon}+\alpha \nabla^{2} \bar{\varepsilon}
$$

where $\alpha$ is a material parameter of the dimension of a length squared. Equation (37) is, in fact, obtained from a second order Taylor development of the effective strain in the non local expression (Eq. 31). Hence, we assume that the support of the weight function is very small compared to the spatial variation of the effective strain over the structure analysed. This new expression of the non local effective strain can be substituted in the equations defining the evolution of damage in section 5.2. Numerically, this constitutive model can be very awkward to implement in a finite element model because Eq. (37) is a differential equation which involves the third order derivatives of the displacement functions. To obviate this problem, Eq. (37) can be approximated by

$$
\bar{\varepsilon}-\alpha \nabla^{2} \bar{\varepsilon} \approx \bar{\varepsilon}
$$

which is much easier to implement in a finite element code as it does not involve more than the first order derivatives of the displacement (see Peerlings et al. 1995).

In the non local and gradient models discussed above, the internal length is postulated, as the result of interactions between growing defects which are, by definition, of a non local character. In some situations, however, it is possible to derive such a model with an internal length from existing, but more advanced, theories of elasticity. These theories are directly 
related to the multiple field theories described by Mariano and Trovalusci (1999) and recalled in the next chapter of this book.

Let us consider a material which contains voids with isotropic characteristics. In fact, we consider a porous isotropic material whose porosity is growing as damage, defined as a scalar quantity, develops. From the micromechanical point of view, damage will be characterised by the variation of volume fraction of material denoted as $v(x, t)$. For a strain free material, the volume fraction is equal to 1 initially, and it decreases when damage grows. Failure is reached when the volume fraction is equal to zero. Starting from a reference configuration where the material is strain - free and the volume fraction is $v_{R}(x)$, the variation of the volume fraction of material is $\phi(x, t)=v(x, t)-v_{R}(x)$. This variation of volume fraction of material can be due to damage growth or straining.

For constant damage, the porous material is elastic and its behaviour is modelled using the theory of elastic material with voids (Cowin and Nunziato, 1983). The governing equations are (in the absence of body forces):

$$
\begin{aligned}
& \sigma_{i j . j}=0 \\
& h_{i . i}+g=0
\end{aligned}
$$

where $h_{i}$ is the equilibrated stress vector, $g$ is the equilibrated body force, and $\sigma$ is the overall stress in the porous material. Equation (39b) was first suggested in the case of granular materials by Goodman and Cow in (1972), and arises also in the microstructural theories of elastic materials. Variables $g$ and $h_{i}$ have been given specific interpretation: they are related to the stresses due to centres of dilation made of three couples of opposite forces without moments acting along three mutually orthogonal directions at material points. Such forces correspond to the local pressures necessary to augment the size of an existing void, in a reversible or irreversible manner and they create a local stress distribution. These forces produce void growth and a variation of the overall volume fraction of the body.

The elastic (free) energy reads:

$$
W=\frac{1}{2} C_{i j k l} \varepsilon^{e} \varepsilon_{k l}^{e}+\beta \delta_{i j} \varepsilon_{1 j}^{e} \phi+\frac{1}{2} \alpha \phi_{. i} \phi_{. i}+\frac{1}{2} \xi \phi^{2}
$$

where $C_{i j k l}$ are the overall stiffness coefficients of the porous material, and $(\alpha, \beta . \xi)$ are material parameters. The above form of the elastic energy is the result of the assumption that the material is isotropic with a centre of symmetry. The thermodynamic forces associated to the variables $\left(\varepsilon_{i j}, \phi, \phi_{. i}\right)$ are defined as :

$$
\sigma_{i j}=\frac{\partial W}{\partial \varepsilon_{i j}} \cdot g=-\frac{\partial W}{\partial \phi}+g_{R}, h_{i}=\frac{\partial W}{\partial \phi_{. i}}+h_{i}^{R}
$$

where $g_{R}$ and $h_{i}{ }^{R}$ are the values of the equilibrated body forces and equilibrated stress vector in the reference state, respectively. Hence equilibrium in the reference (stress-free) state yields: 


$$
h_{i, i}^{R}+g_{R}=0
$$

The constitutive relations are:

$$
\begin{aligned}
& \sigma_{i j}=\lambda \varepsilon_{k i}^{e} \delta_{i j}+2 \mu \varepsilon_{i j}^{e}+\beta \phi \delta_{i j}, \\
& h_{i}=\alpha \phi_{. i}+h_{i}^{R}, \\
& g=-\xi \phi-\beta \varepsilon_{k i}^{e}+g_{R},
\end{aligned}
$$

where $\delta_{i j}$ is the Kronecker symbol.

As pointed out by Cowin and Nunziato, the stiffness coefficients and the material parameters should depend on the reference volume fraction. In order to obtain an extension of this model to the case of a damaged material, it is assumed that whenever damage grows, it modifies the volume fraction of the material in the reference configuration considered by the linear elastic theory. The variation of volume fraction is rewritten as:

$$
\begin{gathered}
d \phi(. x, t)=d \phi^{r e v}(x, t)+d \phi^{i r}(x, t), \\
\text { with }\left\{\begin{array}{l}
\phi^{r e v}(x, t)=v(x, t)-v^{R}(x, t), \\
\phi^{i r}(x, t)=v^{R}(x, t)-v^{0}(x),
\end{array}\right.
\end{gathered}
$$

where $\phi^{r e v}$ is the reversible variation of volume fraction, $v^{R}(x, t)$ is the volume fraction of the damaged material when it is free of loads, $\phi^{i r}$ is the irreversible variation of volume fraction due to the growth of damage measured when the material is free of loads and $v^{0}$ is the initial volume fraction of the material, when damage is equal to zero. The free energy of the material is now rewritten with the introduction of the degradation of the stiffness. Therefore, we assume that $g_{R}$ and $h_{i}^{R}$ defined in the elastic theory are related to $\phi^{i r}$ (Pijaudier-Cabot and Burlion, 1996).

A "source term" $A$ which controls the irreversible variation of volume fraction of the material from the initial state is defined with a loading function $f\left(\varepsilon_{i j}^{e}, Z\right)$ and an evolution equation. It is added to the right hand-side term in Eq. (39b). $Z$ is the hardening - softening variable. For the sake of simplicity, we assume now that $\phi^{r e 1}$ is very small compared to $\phi^{i r}$ as damage grows. We will use, in the following, a linear relation between damage and the irreversible variation of volume fraction : $d=-\phi^{i r}$. The constitutive relations which follow from these assumptions are similar to those of the gradient damage model. The governing equations and the stress - strain relation are now: 


$$
\begin{aligned}
& \left\{\begin{array}{l}
\sigma_{i j . j}=0, \\
\alpha d_{. i i}-\xi d=A,
\end{array}\right. \\
& \sigma_{i j}=(1-d)\left[\lambda \varepsilon_{k i}^{e} \delta_{i j}+2 \mu \varepsilon_{i j}^{e}\right]-\beta \delta_{i j} d .
\end{aligned}
$$

In this final form, we may interpret the different material parameters. $\xi$ is related to the evolution law of damage, in an integrated form and the coefficient $\beta$ controls the amount of volumetric irreversible strain due to damage.

For the finite element implementation, consider now a finite body denoted as $\Omega$ with boundary $\partial \Omega$. This body is initially at rest, the displacement and damage velocities being equal to zero. The two governing equations of motion are those defined in Eqs. $(45 \mathrm{a}, \mathrm{b})$ and the boundary and initial conditions corresponding to damage equation are those defined in the linear elastic theory :

$$
\left\{\begin{array}{c}
u_{i}(x, t)=\tilde{u}_{i}(x, t) \text { on } \partial \Omega_{1} \text { and } \sigma \cdot \vec{n}=\vec{\tau} \text { on } \partial \Omega_{2}, \\
\left(\partial \Omega_{1} \cup \partial \Omega_{2}=\partial \Omega \text { and } \partial \Omega_{1} \cap \partial \Omega_{2}=\varnothing\right) \\
\alpha \operatorname{grad}(d(x, t)) \cdot \vec{n}=0 \text { on } \partial \Omega
\end{array}\right.
$$

where $\vec{n}$ is now the unit outward normal to the boundary $\partial \Omega$. The meaning of the third (natural) boundary condition in Eq. (46) remains a problem to be solved.

In the absence of body forces and irreversible strains, the two governing equations, along with the boundary conditions, are equivalent to the following conditions: find a displacement field $\vec{u}$ and a damage field $d$ such that for any cinematically admissible virtual displacement $\vec{u} *$ and damage field $d^{*}$ :

$$
\begin{aligned}
& \int_{\Omega} \varepsilon^{*}: \sigma d v=\int_{\partial \Omega} \vec{u}^{*} \cdot \vec{F} d s, \\
& \int_{\Omega}\left\{\alpha \operatorname{grad}\left(d^{*}\right) \cdot \operatorname{grad}(d)+\xi d^{*} d\right\} d v=\int_{\Omega} d^{*} \cdot A d v,
\end{aligned}
$$

where $\vec{F}$ are external applied forces. For the finite element implementation, the displacement field components and damage field are discretised, same as in coupled thermo-mechanical problems. A similar variational principle can be obtained in an incremental fashion for the purpose of implementing the model within a Newton - Raphson procedure. 


\subsection{Analysis of Strain and Damage Localisation}

Throughout this section, we are going to investigate the properties of the non local damage model when strain and damage localisation occurs. For the sake of simplicity, we will use the energy-based damage model. The strain-based model is certainly closer to the behaviour of concrete and rock-like materials. It is much more complex from a mathematical point of view (because of the expression of the equivalent tensile strain which involves positive parts) and exhibits similar properties which have been verified numerically. The bifurcation analyses which are presented hereafter aim at understanding the properties of the non local model and the influence of the internal length.

\subsubsection{One Dimensional Approach to Localisation}

For the sake of simplicity we will consider first the one dimensional case of a bar which is in an initial state of constant strain $\varepsilon$. The stress-strain curve of the material is shown in Fig. 1 la. It exhibits strain softening with a constant modulus $E_{t}$ and a secant modulus denoted as $E_{u}$. The length of the bar is $L$ and its cross section is $A$.
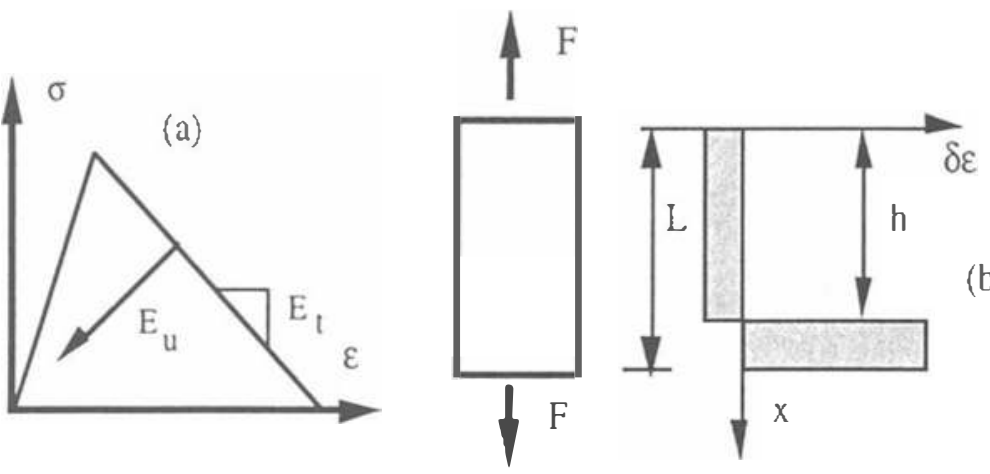

Figure 11. Localisation in the one dimensional case

Assume that the loading is displacement controlled. If the strain $\varepsilon=u / L$ is lower than the strain at peak stress, the stress in the bar is $\sigma=E_{u} \varepsilon$. For initial states of strain beyond this limit, the solution of the equation of equilibrium to small stress perturbation is not unique.

Assume now that for $\mathrm{x} \in[0, \mathrm{~h}]$, the incremental stiffness is $E_{u}$ and that for $\left.\mathrm{x} \in \mathrm{h}, \mathrm{L}\right]$ the incremental stiffness is $E_{t}$. It is then possible to find a possible perturbation, solution to this problem for the constitutive and compatibility equations are: 


$$
\begin{aligned}
& \begin{cases}d \sigma=E_{u} d \varepsilon_{u} & \text { for } x \in[0, h] \\
d \sigma=E_{t} d \varepsilon_{t} & \text { for } x \in] h, L]\end{cases} \\
& h d \varepsilon_{u}+(L-h) d \varepsilon_{t}=d u
\end{aligned}
$$

If the variation of the displacement at the extremity of the bar is fixed, the corresponding variation of tensile load is:

$$
d F=A\left[\frac{E_{u} E_{t}}{\left(h E_{t}+E_{u}(L-h)\right)}\right] d u
$$

Hence, for any value of the unknown $h$, it is possible to find a solution to this problem. The number of solutions for this problem is infinite. It depends on the size of the zone where the. strain is increasing incrementally, and also on its location.

Under displacement controlled conditions, the second order work corresponding to the perturbation is:

$$
d^{2} U=\frac{1}{2} \int_{0}^{L} d \varepsilon \cdot d \sigma d x-d F d u
$$

Upon substitution of the constitutive relations and of Eq. (49), the following form of the second order work is:

$$
d^{2} U=-\frac{1}{2} A E_{t}\left(d \varepsilon_{t}\right)^{2}\left(\frac{h E_{t}}{E_{u}}+(L-h)\right)
$$

It follows that the state of equilibrium from which the perturbation is considered is stable if

$$
\left(\frac{h E_{t}}{E_{u}}+(L-h)\right)>0
$$

and stability is directly a function of the size of the region in which the strain increases incrementally.

If this size is not controlled by the constitutive relations, the stable solution will be the one that minimises the second order work, according to Gibbs definition of stability. A simple calculation shows that the stable solution is obtained for $h \rightarrow L$. In this case, all the bar unloads except for one point which follows the softening curve. This is the one dimensional localisation mode in which the admissible incremental strain is discontinuous. As a consequence, the energy dissipation is confined to a point (region of zero volume) and the total energy dissipated by the bar during failure is zero (the dissipation per unit volume of 
material is finite). Obviously, this result is not realistic. It is also in contradiction with crack propagation criteria (such as Griffith criterion).

The localisation mode studied in this example is possible because of strain softening. It cannot occur in the hardening regime because it is not possible then to find a perturbation of the form in Eq. (48) which satisfies equilibrium (the incremental strain has to be constant over the bar).

Let us consider now the case of a non local damage model. In order to avoid the difficulties inherent to the boundary conditions and the local averaging procedure near the boundaries, we look at an infinite bar which is in a homogeneous state of stress, strain, and damage initially denoted as $\left(\sigma_{0} \varepsilon_{0}, D_{0}\right)$. The rate equation of equilibrium is derived from Eqs. $(8,9,24,25)$, assuming that the variation of stress is homogeneous over the bar:

$$
\left(1-D_{0}\right) E_{0} \dot{\varepsilon}_{x}-F(\bar{y}) \frac{1}{V_{r}(x)} \int \psi(s)\left(E_{0} \varepsilon_{0}\right)^{2} \dot{\varepsilon}(x+s) d s=0
$$

where $E_{0}$ is the elastic constant of the material. Note that the representative volume $V_{r}(x)$ is constant for an infinite bar and that in this problem, the increments are replaced by rates, which is the same since the constitutive relations are rate independent.

Instead of being a differential equation, the equation of equilibrium is now a second order linear integral equation in $\dot{\varepsilon}_{, x}$. Thus, the mathematical nature of the problem is slightly changed. Nevertheless, it is possible to recover an algebraic equation by considering harmonic solutions of the velocity field $i(x)$. This means that possible solutions are developed in Fourier series. This type of solution enables to separate, in the kernel of the integral, variable $x$ from variable $s$. Hence, the kernel is degenerated in order to transform the integral equation into an algebraic equation. For details the reader should consult the book by Courant and Hilbert (1953).

$$
\dot{u}(x)=A \exp (-i \xi x)
$$

where $i$ is now the imaginary constant such that $i^{2}=-1$. Substitution in the equation of equilibrium yields:

$$
A \xi \exp (-i \xi \xi)\left[\left(1-D_{0}\right) E_{0}-F(\bar{y}) \hat{\psi}\left(\xi, l_{c}\right)\left(E_{0} \varepsilon_{0}\right)^{2}\right]=0
$$

with

$$
\hat{\psi}\left(\xi, l_{c}\right)=\exp \left(-\frac{\xi^{2} l_{c}^{2}}{2}\right)
$$

$\hat{\psi}\left(\xi, l_{c}\right)$ is the Fourier transform of the weight function. There is a trivial solution to this equation where $\xi$ is zero and the strain remains homogeneous over the bar. However, another solution may exist: 


$$
\xi^{2}=-\frac{2}{l_{i}^{2}} \ln \left(\frac{\left(1-D_{0}\right)}{E_{0} \varepsilon_{0}^{2} F(\bar{y})}\right) \text { if }\left[\left(1-D_{0}\right)-E_{0} \varepsilon_{0}^{2} F(\bar{y})\right]<0
$$

The condition of existence of such a solution coincides with the condition that the tangent modulus of the material deformed homogeneously be negative or zero. Thus, loss of uniqueness is possible starting at the peak stress and beyond, in the strain-softening regime. The wave length of the periodic solution is proportional to the characteristic length of the material and depends on the initial state of strain (or tangent modulus) of the bar. In this simple case, it can be seen that the number of possible solutions of the rate equation of equilibrium is restricted to the trivial (homogeneous) solution and to a periodic solution. If the constitutive equations are local $\left(l_{c}=0\right)$, there is an infinite number of solutions to this problem, same as in the previous paragraph along with the same unrealistic feature of zero energy dissipation at failure.

With the gradient damage model developed in section 6, the system of equations of equilibrium reduces to:

$$
\begin{aligned}
& E_{0}\left(1-d_{0}\right) \dot{\varepsilon}_{. x}-\left(E \varepsilon_{0}+\beta\right) \dot{d}_{, x}=0 \\
& \alpha \dot{d}_{. x x}-\xi \dot{d}=\dot{A}
\end{aligned}
$$

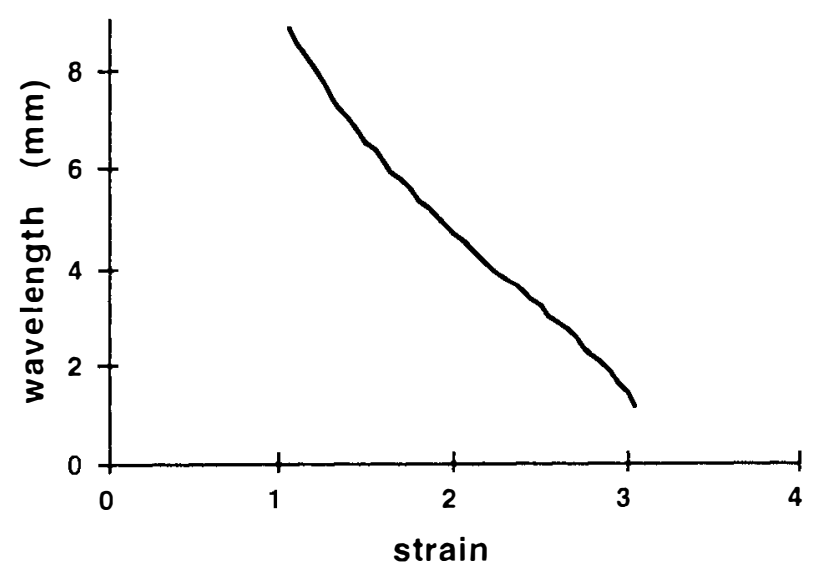

Figure 12. Simplified one dimensional damage model: wave length of the localised mode as a function of the strain at the onset of bifurcation.

We are using here a evolution of damage such that in the softening regime, the material behaves linearly with a tangent modulus denoted as $h$. Harmonic solutions are found upon softening $(h<0)$ only. The wave lengths $\ell$ of these solutions are function of the initial state of damage and of the material parameter $\alpha$ : 


$$
\ell=2 \pi \sqrt{-\frac{\alpha E_{0}\left(1-d_{0}\right)}{\xi h}}
$$

Bifurcation occurs when softening is encountered. Besides the trivial solution where the strain and damage rates remain homogeneous, there is again a second possible solution to the governing equations of equilibrium when the material softens (the term under the square root sign in Eq. (59) must be positive).

The square root of $\alpha$ is an internal length of the continuum which selects the wave length of the localised solution and scales the size of the localisation band. It plays exactly the same role as the internal length in other localisation limiters (see e.g. Sluys 1992, Pijaudier-Cabot and Benallal 1993). Figure 12 shows the variation of the wave length as a function of the initial strain $\varepsilon^{0}$ about which bifurcation is considered. The material parameters are $E=1 \mathrm{MPa}, h=-0.5 \mathrm{MPa}, \xi=1, \beta=0.1 \mathrm{MPa}$, peak stress $=1 \mathrm{MPa}$ with $\alpha=1 \mathrm{~mm}^{2}$.

\subsubsection{Possible Localisation Modes in Static's}

We will consider now the case of an infinite solid on which adequate boundary conditions (rate of loading) are applied at infinity. It is assumed that the solid is initially deformed homogeneously and that it is subjected to a quasi-static increment of deformation. For any variation about the initial equilibrium state, the rate equations of equilibrium are:

$$
\begin{aligned}
& \dot{\sigma}(x)=\left(1-D_{0}\right) E: \dot{\varepsilon}(x) \\
& -\frac{F\left(\bar{y}_{0}\right) E: \varepsilon_{0}(x)}{V_{r}(x)} \int_{V} \psi(s) \varepsilon_{0}(x+s): E: \dot{\varepsilon}(x+s) d s
\end{aligned}
$$

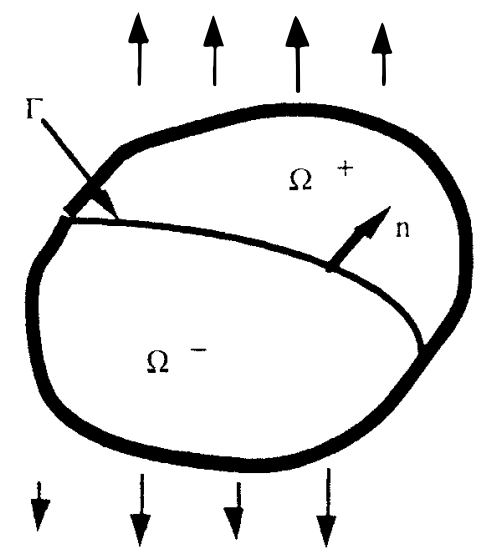

Figure 13. Solid with a discontinuity of the strain rate field through the surface $\gamma$. 
The subscript 0 in this equation denotes the initial state of deformation and damage from which the perturbation are considered. Following Rudnicki and Rice analysis (1975). the velocity field corresponding this perturbation is assumed to be $\mathrm{C}_{0}$-continuous while the corresponding strain rate is $\mathrm{C}_{-1}$-continuous, discontinuous across a surface denoted as $\Gamma$ with normal vector $\bar{n}$ (Fig. 13).

For equilibrium, the stress rate jump derived from the assumed strain distribution across $\Gamma$ must be zero:

$$
[\dot{\sigma}] \cdot \vec{n}=\overline{0}
$$

where $[\dot{\sigma}]$ is the stress rate jump, that is the difference between the stress rate tensors across surface $\Gamma$. In order to proceed with the analysis, it is necessary to know what are the loading conditions in the two parts of the solid separated by $\Gamma$. A part from the trivial case in which the two parts unload and the strain rate jump is zero, it may be assumed that one part of the solid denoted as $\Omega^{-}$unloads (damage is constant according to Eq. 24) while damage keeps on growing in the other part denoted as $\Omega^{+}$. The average damage energy release rate at any point of the discontinuity surface is, however, a $\mathrm{C}_{0}$-continuous function :

$$
\dot{\bar{y}}(x)=\frac{1}{V_{r}(x)} \int_{V^{\prime}} \psi(s) \mathcal{E}_{0}(x+s): E: \dot{\varepsilon}(x+s) d s
$$

Thus, a discontinuity of the damage growth condition is impossible on $\Gamma$, the only remaining possibility for a discontinuity of the strain rate field to exist is that damage should grow in $\Omega^{-}$ and in $\Omega^{+}$and the damage jump across the discontinuity surface $\Gamma$ should be zero according to Eqs.(25, 62). Therefore, the discontinuity of the normal stress across the interface is proportional to the strain rate jump. It can be easily checked that this jump must be zero in order to satisfy equilibrium (Eq. 61). Consequently, a discontinuous strain rate field cannot be an equilibrium solution according to the non local model. A similar property is trivial for gradient models, since the displacement field must be continuous enough so that second order gradients of the strain exist.

\subsubsection{Three Dimensional Analysis with the Non Local Model}

Strain localisation cannot be defined as the onset of a discontinuity of the velocity field. It remains however that strain softening may produce a loss of uniqueness of the solution to a boundary conditions problem. The detection of bifurcation points cannot be carried out analytically in the general context. This analysis can be perfomed in the case of an infinite body only, assuming that the boundary conditions are such that the deformation and damage variables are initially homogeneous over the solid. Hence, we will study the conditions of uniqueness and admissibility of small perturbations which satisfy the rate constitutive equation (60) and the momentum equation: 


$$
\operatorname{div}(\dot{\sigma}(x))=\rho \frac{\partial^{2} \dot{\vec{u}}(x)}{\partial t^{2}}
$$

where $\rho$ is the mass density and $\dot{\vec{\imath}}$ is the unknown perturbation. We will consider here the non local damage model only. Similar results can be obtained with the gradient damage model.

With the non local model, Eq. (63) is an integro-differential equation. Solutions of this equations are harmonic waves, propagating in direction $\vec{n}$, of amplitude $\vec{A}$ and phase velocity $c$ :

$$
\dot{\vec{u}}(x)=\vec{A} \exp (-i \xi(\vec{n} \cdot \vec{x}-c t))
$$

Substitution of these solutions into the equation of motion yields the linear algebraic system:

$$
\left(\vec{n} \cdot H^{*}(\xi) \cdot \vec{n}-\rho c I\right) \vec{A}=\overrightarrow{0}
$$

where $I$ is the $2 \times 2$ identity matrix, $H^{*}(\xi)$ is the tangent operator governing the strain rate stress rate relation and:

$$
\begin{aligned}
\vec{n} \cdot H^{*}(\xi) \cdot \vec{n}= & \left(1-D_{0}\right) \vec{n} \cdot E \cdot \vec{n} \\
& -\hat{\psi}(\xi) F\left(\bar{y}_{0}\right)\left(E: \varepsilon_{0} \cdot \vec{n}\right) \otimes\left(E: \varepsilon_{0} \cdot \vec{n}\right)
\end{aligned}
$$

where $\otimes$ denotes the tensorial product. $\vec{n} \cdot H^{*}(\xi) \cdot \vec{n}$ can be regarded as a pseudo acoustic tensor, function of the wave number $\xi$ entering in $\hat{\psi}(\xi)$ which is the Fourier transform of the weight function:

$$
\hat{\psi}(\xi)=\exp \left(-\frac{\xi^{2} l^{2}}{2}\right)
$$

The condition of bifurcation, i.e. the condition of admissibility and non uniqueness of harmonic waves is (a homogeneous deformation with constant velocity is already a trivial solution):

$$
\operatorname{det}\left[\vec{n} \cdot H^{*}(\xi) \cdot \vec{n}-\rho c I\right]=0
$$

The major difference with the same analysis performed on a local continuum is that the pseudo-acoustic tensor is a function of the wave number. In a local damage model, the weight function is a Dirac function. The Fourier transform of such a function is constant. Whenever Eq.(68) is satisfied for a local continuum, there is an infinity of possible solutions corresponding to any arbitrary wave number. On the contrary, Eq.(68) may be satisfied in a non local continuum for a limited number of values of $\xi$. 
In order to exhibit the localisation conditions in static's, the phase velocity in Eq.(64) is taken equal to zero. Eq.(69) is the bifurcation condition:

$$
\operatorname{det}\left[\vec{n} \cdot H^{*}(\xi) \cdot \vec{n}\right]=0
$$

It has the same form as Rudnicki and Rice condition (1975). In the limit of a vanishing internal length, Eq. (69) is exactly Rudnicki and Rice expression which corresponds to the occurrence of a surface of discontinuity of the strain rate in the considered solid. The acoustic tẻnsor $\bar{n} H \bar{n}$ is singular.

The relation between the singularity of the acoustic tensor in a continuum description and the progressive concentration of failure in discrete analysis is rather difficult to apprehend. A first reason is that it can be quite problematic to derive a tangent operator $H$ in discrete analyses. Delaplace et al. (1999) have recalled that in some situations at least (e.g. for a discrete interface), the characteristic of the system (force vs. displacement) is not derivable. A second reason is that due to the initial disorder, bifurcation is very seldom to occur. The initial disorder serves as a perturbation which triggers damage localisation. According to the above analysis, the bifurcation condition is derived assuming that the system is initially in a homogeneous state of deformation. Would/bifurcation be permitted in discrete analyses, responses of systems without localisation of fracturing events should be observed. It is from such results that an equivalent tangent operator of the underlying continuum approximation of discrete system should be computed, before strain localisation, same as in Rudnicki and Rice analysis. This type of result has never been obtained to our knowledge. Hence, the determinant of the acoustic tensor, computed from an averaged response of several discrete analyses where a progressive concentration of damage occurs, should not vanish. At best, it should decrease

without becoming singular. This type of result has been observed by Krajcinovic and Vujosevic (1998).

\subsection{Finite Element Example}

The non local model is relatively easy to implement since the equilibrium equations are standard. The integral relation due to the non local term is discretised according to the finite element mesh used for the analysis and an usual quadrature rule is employed for its evaluation. Notice that the weight function does not vary during the loading process and is independent of the boundary conditions. Therefore, the numerical evaluation of the non local terms is simplified: prior to initiate the calculations, the average weights are computed at each integration point and stored once and for all. In finite element calculations, the weight function is chopped off: the weights that are less than 0.001 are set to zero. The actual volume of integration does not span over the entire volume of the solid and the calculation of the integrals requires less computer time and memory as the number of neighbouring integration points is reduced.

Figure 14 shows a schematic problem statement (see e.g. Pijaudier-Cabot and Bazant (1987) for details). A constant velocity is applied at the extremities of the bar such that a constant strain wave of amplitude $0.7 \varepsilon^{*}$ (where $\varepsilon^{*}$ is the strain measured at the peak stress) is propagated. These waves meet at $t^{*}=1 \mathrm{~s}$ in the middle of the bar where localisation occurs. 


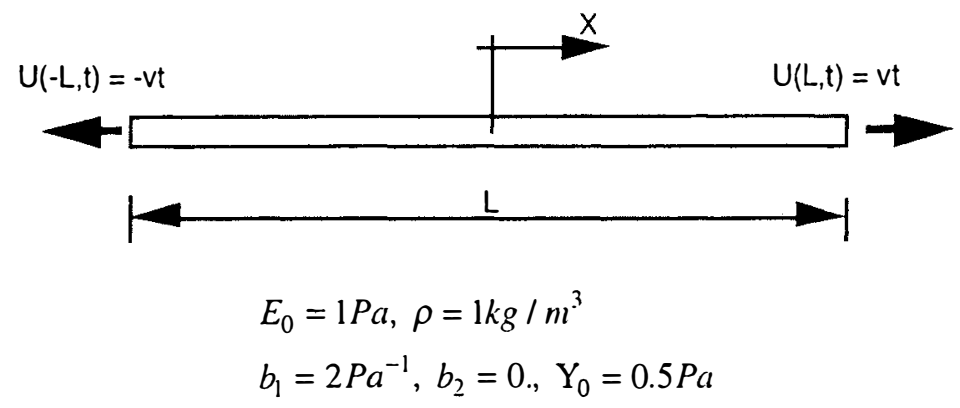

Figure 14. Dynamic bar problem.

Figure 15 shows the results of computations carried out with a local model (internal length set to zero) and several meshes with constant length elements. The damage profiles are plotted on this figure at time $1.5 \mathrm{t}^{*}$.

Damage localises into the two elements at the middle of the bar. The size of the damaged zone is controlled by the size of the finite elements: the smaller the element size, the smaller the damage zone will be. At the limit of a vanishing element size, which should correspond to the solution for a continuum, damage localises over a segment of zero length. Since the energy dissipation per unit volume of material is finite, the total energy dissipation for the entire bar, defined as the integration over time and over the bar of the dissipation rate, tends to zero. Failure occurs without energy dissipation, which is not physically realistic.

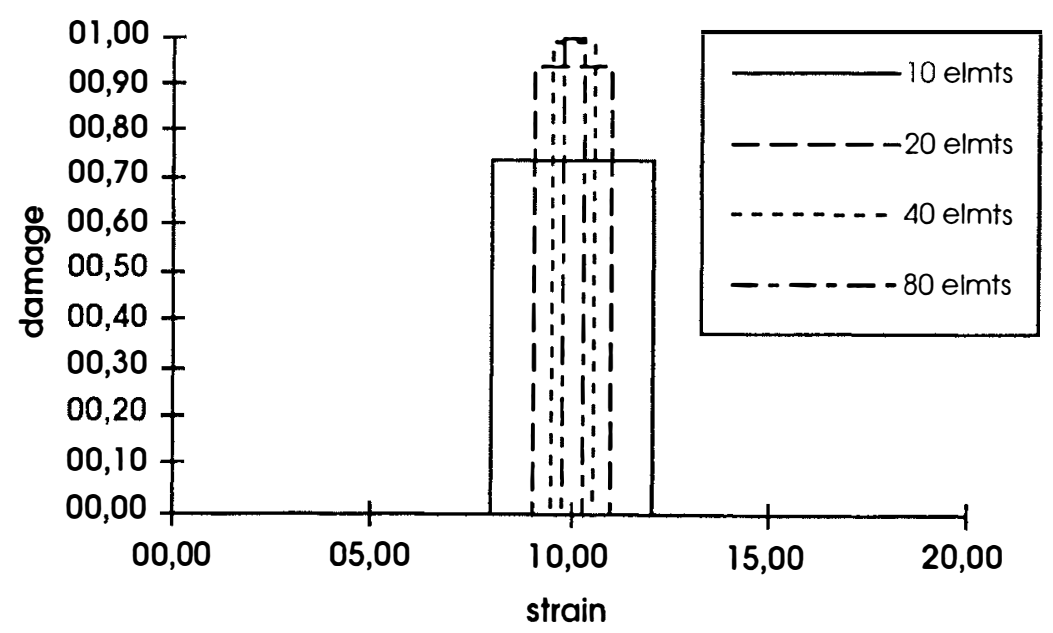

Figure 15. Local damage model - damage profiles for meshes with constant length element. 
Figure 16 shows the same calculations performed with the non local model. Upon mesh refinement, the profiles of damage remain constant. The size of the damaged zone is proportional to the internal length of the continuum and failure occurs with a finite. non zero energy dissipation because damage localises over a portion of the bar of finite, non zero length.

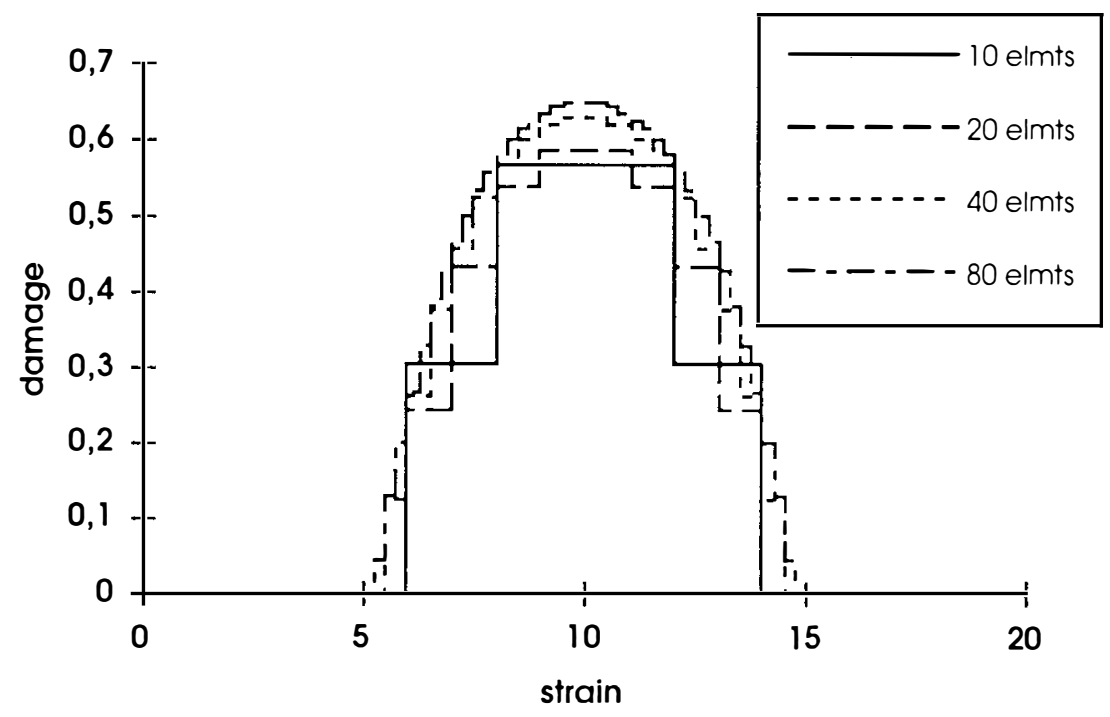

Figure 16. Non local damage model - damage profiles for meshes with constant length element.

\subsection{Relation with Fracture Mechanics}

Fracture mechanics and its link with damage mechanics may possibly help at understanding the effect of non locality of damage and the influence of the internal length on macroscopic fracture properties. Our objectives in this section is to discuss a very simplified link between the two theories which exhibit the influence of the internal length of the material and may possibly be used for approximating this length experimentally.

Consider an infinite body subjected to uniaxial tension in direction 2. $\sigma_{22}^{0}$ with $\sigma_{i j}^{0}=0$ for $i \neq 2$ and $j \neq 2$. Damage is denoted as $D_{0}$ and the strains are denoted as $\varepsilon_{i j}^{0}$. When small deviations from this equilibrium state are analysed, harmonic displacement fields are solutions of the partial differential equations of equilibrium given in section 7 .

We assume now that damage localisation is such that the band is perpendicular to the tensile load (Fig. 17). The wave length of the harmonic solution is entirely determined from the evolution law of damage and the internal length of the continuum because the orientation of the band is fixed. The calculation of the approximated fracture energy is based on the assumption that at the onset of strain localisation, i.e. at the onset of localised craching, the distributions of strain and damage jump suddenly from a homogeneous distribution to a 
harmonic solution with the smallest possible wave length. This is based on stability considerations which require that the width of the localisation zone should be as small as possible and on the assumption that intermediate states where maximum damage is neither equal to zero nor to 1 have a relatively small influence on the energy dissipation.

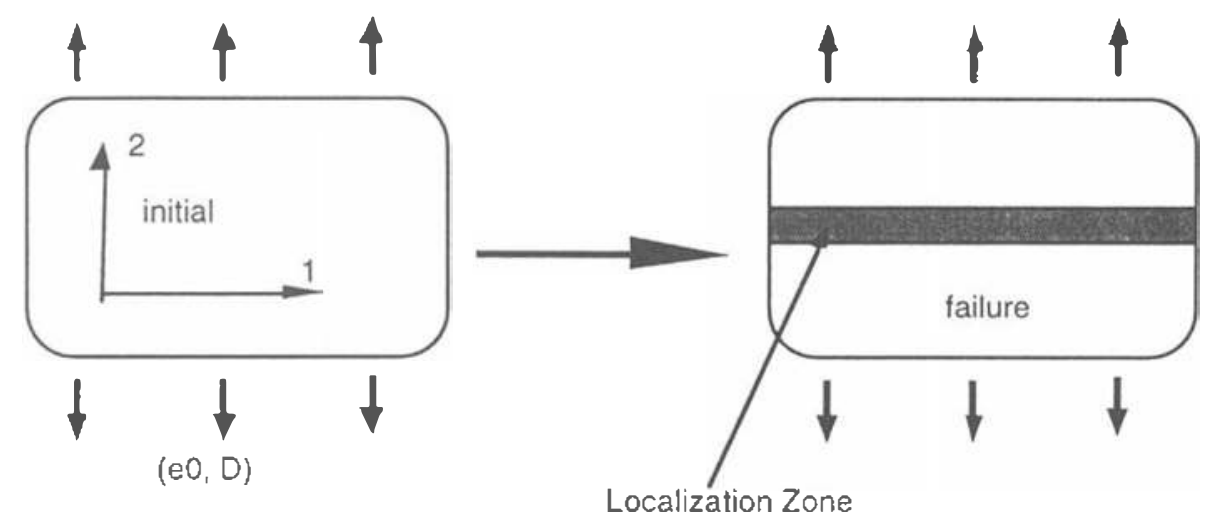

Figure 17. Geometry of the localisation band assumed for the calculation of the fracture energy.

In an infinite body, localisation occurs suddenly and maximum damage jumps very rapidly to one. Therefore, this assumption does not seems to be too far away from the exact process of localisation in which the region where damage evolves shrinks with increasing damage as the loading progresses. Furthermore, this approximation has been already tested successfully in finite element analyses of pre-damaged structures in order to derive an equivalence between a cracked and a damaged (in the continuum sense) structural component (Bodé et al. 1997). This equivalence was derived with the strain based damage model developed in section 5 .

With the minimum wave length (maximum value of $\xi$ in Eq. (64)), the distribution of damage perpendicularly to the crack direction (the coordinate is denoted as $x_{2}$ ) is:

$$
\begin{gathered}
D\left(x_{2}\right)=\frac{\int_{-\infty}^{+\infty} \psi(s) \eta\left(x_{2}+s\right) d s}{\int_{-\infty}^{+\infty} \psi(s) \eta(s) d s} \\
\text { with } \eta(z)=\cos \left(\xi_{\max } z\right) \text { if } z \in\left[\frac{-\pi}{2 \xi_{\max }}, \frac{\pi}{2 \xi_{\max }}\right] \\
\qquad \eta(z)=0 \text { elswhere }
\end{gathered}
$$

The energy consumption due to crack propagation is the integral of the energy dissipation at each material point of coordinate $x_{2}$ in the fracture process zone which encountered damage up to $D\left(x_{2}\right)$. For propagation of a crack over a length $\delta a$, the formula reads 


$$
G_{f} \delta l=\int_{-\infty}^{+\infty} W\left(D\left(x_{2}\right)\right) d x_{2} \delta l
$$

where $W(D)$ is the energy dissipation due to damage up to the value $D$

$$
W\left(D\left(x_{2}\right)\right)=\int_{0}^{D\left(x_{2}\right)} \frac{1}{2} E \varepsilon_{22}^{2}(D) d D=\int_{0}^{\varepsilon_{22}\left(x_{2}\right)} \frac{1}{2} E \varepsilon_{22}^{2} F(\bar{y}) d \varepsilon_{22}
$$

Therefore, the fracture energy is:

$$
G_{f}=\int_{-\infty}^{+\infty} \int_{0}^{\varepsilon_{22}\left(x_{2}\right)} \frac{1}{2} E \varepsilon_{22}^{2} F(\bar{y}) d \varepsilon_{22} d x_{2}
$$

Note that the width of the fracture process zone is implicitly fixed by equation (70). The fracture energy for an infinitely large specimen is related to the model parameters in the constitutive relations including the internal length. For a local model, the wave length in Eq.(70) would be zero and the calculation would yield a zero fracture energy' too. This is not consistent with fracture mechanics where a finite amount of energy is required in order for a crack to propagate. The non local model provides a consistent result via the introduction of an internal length which scales the localisation band (wave length of the localised mode).

\subsection{Conclusions}

Continuous damage mechanics is a theory which aims at describing the mechanical effect of cracking and void growth in an elastic material. Analyses on lattices show that in phenomenological models, the variation of the material stiffness is the appropriate damage variable. It shows also that in the course of failure spatial correlation develops which can be transposed in a non local format in macroscopic models. The description is essentially phenumenological although it may be also motivated by several micro mechanics analyses.

Non locality introduces an internal length which scales the localisation process. In static's, the internal length controls the minimum wave length of the localisation modes. For a rate independent continuum, this property is central and insures a proper convergence of the finite element calculations toward a unique solution or a finite number of solutions analogous to buckling modes. Finally, the internal length makes it possible to relate damage models to fracture mechanics. The fracture energy is function of this internal length. This last parameter could therefore be determined experimentally from fracture tests, using inverse analysis techniques. 


\subsection{References}

Bazant, Z.P. (1985). Mechanics of Distributed Cracking. Applied Mechanics Review 39: 675705.

Bazant, Z.P. (1987). Why Continuum Damage is Non Local: Justification by Quasi-Periodic Microcrack Array. Mechanics Research Communications 14: 407-419.

Bazant, Z.P. and Pijaudier-Cabot, G. (1998). Non local Continuum Damage, Localisation Instability and Convergence. Journal of Applied Mechanics ASME 55: 287 - 294.

Bazant, Z.P. and Pijaudier-Cabot, G. (1989). Measurement of Characteristic Length of Non local Continuum. Journal of Engineering Mechanics ASCE 115: 755-767.

Benallal, A., Billardon, R. and Geymonat, G. (1988). Some Mathematical Aspects of the Damage Softening Rate Problem. Cracking and Damage. J. Mazars and Z.P. Bazant Eds., Elsevier Pubs., 247-258.

Bode, L., Tailhan, J.L., Pijaudier-Cabot, G., La Borderie, C. and Clément, J.L. (1997). Failure Analysis of Initially Cracked Concrete Structures. Journal of Engineering Mechanics ASCE 123: 1153-1160.

Chudnovsky, A., Botsis, J. and Kunin, B. (1989). The Role of Microdefects in Fracture Propagation Process. Cracking and Damage. J. Mazars and Z.P. Bazant Eds., Elsevier Pubs., 140-149.

Courant R. and Hilbert D. (1953). Methods of Mathematical Physics. Vo. 1, Interscience Pubs.

Cowin, S.C. and Nunziato, J.W. (1983). Linear Elastic Materials with Voids. Journal of Elasticity 13: 125-147.

De Arcangelis, L. and Herrmann, H.J. (1989). Scaling and Multiscaling Laws in Random Fuse Networks. Physical Review B 39: 2678-2684.

Delaplace, A., Pijaudier-Cabot, G., and Roux, S. (1996). Progressive Damage in Discrete Models and Consequences on Continuum Modelling. Journal of the Mechanics and Physics of Solids 44: 99-136.

Delaplace, A., Roux, S., and Pijaudier-Cabot, G. (1999). Damage Cascade in a Softening Interface. International Journal of Solids and Structures 36: 1403-1426.

Eringen, A.C. and Edelen, D.G.B. (1972). On Non local Elasticity. International Journal of Engineering Science 10: 233 - 248.

Goodman, M.A., Cowin, S.C. (1972). A Continuum Theory for Granular Materials. Archives of Rational Mechanics Analysis 44: 249.

Kachanov, M. (1987). Elastic Solids with Many Cracks. A Simple Method of Analysis. International Journal of Solids and Structures 23: 23-43.

Krajcinovic, D. (1989), Damage Mechanics. Mechanics of Materials 8: 117-197.

Krajcinovic, D. and Basista, M. (1991). Rupture of Central Force Lattices Revisited. Journal de Physique 1: 241-245.

Krajcinovic, D. and Vujosevic, M. (1998), Strain Localization - Short to Long Correlation Length Transition. International Journal of Solids and Structures 35, 4147.

Laws, N. and Brockenbrough, J.R. (1987). The Effect of Micro crack Systems on the Loss of Stiffness of Brittle Solids. International Journal of Solids and Structures 23: 1247-1268. 
Lemaitre, J. and Chaboche, J.L. (1989). Mechanics of Solid Materials. Cambridge University Press.

Mariano, P.M. and Trovalusci, P. (1999). Constitutive Relations for Elastic Microcracked Bodies: from a Lattice Model to a Muitifield Continuum Description. International Journal of Damage Mechanics 8: 153-173.

Mazars, J. (1984). Application de la mécanique de l'endommagement au comportement non linéaire et à la rupture du béton de structure. Thèse d'état de l'Université Paris VI, France.

Peerlings, R.H., de Borst, R., Brekelmans, W.A.M., de Vrè, J.H.P. (1995). Computational Modelling of Gradient Enhanced Damage for Fracture and Fatigue Problems. Computational Piasticity, D.R.J. Owen, E. Onate, E. Hinton Eds, Pineridge Press, 1: 975986.

Pijaudier-Cabot, G. and Berthaud, Y. (1990). Effets de interactions dans l'endommagement d'un milieu fragiie - formulation nonlocale. Comptes Rendus de l'Académie des Sciences $\mathrm{t}$. 310, II: $1577-1582$.

Pijaudier-Cabot, G. and Bazant, Z.P. (1987). Nonlocal Damage Theory. Journal of Engineering Mechanics ASCE 113: 1512 - 1533.

Pijaudier-Cabot, G. and Bodé, L. (1992). Localisation in a Nonlocal Continuum. Mechanics Research Communications 19: 145-153.

Pijaudier-Cabot, G. and Benallal, A. (1993). Strain Localisation and Bifurcation in a Non local Continuum. International Journal of Solids and Structires 30 1761-1775.

Pijaudier-Cabot, G., Burlion, N. (1996). Damage and Localisation in Elastic Materials with Voids. International Journal of Cohesive Frictional Materials 1: 129-144.

Rudnicki, J.W. and Rice, J.R. (1975). Conditions for the Localisation of Deformation in Pressure Sensitive Dilatant Materials. Journal of the Mechunics and Phy'sics of Solids 23: $371-394$.

Saouridis. C. (1988). Identification et numérisation objectives des comportements adoucissants - une upproche multi-échelle du comportement du béton. Thèse de Doctorat, Université Paris VI, France.

Schmittbuhl. J. and Roux, S. (1994). Influence of Internal Stresses on Fracture of Heterogeneous Media. Modern Simuiations in Material Science Engineering 2: 21-52.

Sluys, L.J. (1992). Wave Propagation, Localisation and Dispersion in Softening Solids, Doctoral Dissertation. Delft University of Technology, The Netherlands.

Valanis, K C. (1991). A Global Damage Theory and the Hyperbolicity of the Wave Problem. Journal of Applied Mechanics ASME 58: 311-316. 